\title{
Differential encoding of safe and risky offers
}

\author{
David J-N. Maisson ${ }^{1}$, Seng Bum Michael Yoo ${ }^{1,2,3}$, Maya Zhe Wang ${ }^{1}$, \\ Tyler V. Cash-Padgett ${ }^{1}$, Jan Zimmermann ${ }^{1}$, and Benjamin Y. Hayden ${ }^{1}$
}

1. Department of Neuroscience, Center for Magnetic Resonance Research, Center for Neuroengineering, Department of Biomedical Engineering University of Minnesota, Minneapolis MN 55455

2. Center for Neuroscience Imaging Research, Institute for Basic Science, Suwon, Republic of Korea, 16419.

3. Department of Brain and Cognitive Sciences, Massachusetts Institution of Technology, Cambridge, Massachusetts, MA, 02139

\section{* Corresponding author}

\section{David J-N Maisson}

Department of Neuroscience, University of Minnesota

Minneapolis, MN, 55455

Email: maiss002@umn.edu

\section{Keywords}

Common currency models, amodal value signal, ventromedial prefrontal cortex, orbitofrontal cortex, pregenual cingulate cortex, core reward regions

\section{Acknowledgements}

We thank Meghan Castagno Pesce, Marc Mancarella, Caleb Strait and Tommy Blanchard for assistance with data collection, Sarah Heilbronner for help with anatomy, and the rest of the Hayden and Zimmermann labs for valuable discussions. This research was supported by a National Institute on Drug Abuse grant P30 DA048742-01A1 (to BYH and JZ), a National Institute for Biomedical Imaging Grant P41 EB027061 (to BYH and JZ), and a UMN AIRP award (to BYH and JZ).

\section{Competing interests}

The authors have no competing interests to declare. 


\section{ABSTRACT}

41 Common currency theories in neuroeconomics hold that neurons in specific brain regions

42 specifically encode subjective values of offers and not stimulus-specific information. The

43 rationale behind these theories is that abstract value encoding lets the decision maker compare

44 qualitatively different options. Alternatively, expectancy-based theories hold that the brain

45 preferentially tracks the relationship between options and their outcomes, and thus does not

46 abstract away details of offers. To adjudicate between these theories, we examined responses of

47 neurons in six reward regions to risky and safe offers while macaques performed a gambling

48 task. In all regions, responses to safe options are unrelated to responses evoked by equally

49 preferred risky options. Nor does any region appear to contain a specialized subset of value-

50 selective neurons. Finally, in all regions, responses to risky and safe options occupy distinct

51 response subspaces, indicating that the organizational framework for encoding risky and safe

52 offers is different. Together, these results argue against the idea that putative reward regions

53 carry abstract value signals, and instead support the idea that these regions carry information that

54 links specific options to their outcomes in support of a broader cognitive map. 


\section{INTRODUCTION}

The idea that the brain makes use of a common currency (sometimes called abstract)

58 value representations has been foundational within neuroeconomics and the neuroscience of

59 reward (Montague \& Berns, 2002; FitzGerald et al., 2009; Kable \& Glimcher, 2009; Padoa-

60 Schioppa, 2011; Levy \& Glimcher, 2012; Gross et. al, 2014; O’Donoghue \& Rabin, 2015).

61 Indeed, the identification of abstract value representation has been identified as the central

62 research problem in the field (Rangel et al., 2008; Kable \& Glimcher 2009; Padoa-Schioppa \&

63 Conen, 2017). A sine qua non of common currency coding is the existence of pure value neurons

64 - neurons whose firing rate encodes the value of an offer on a single scale (Dorris \& Glimcher,

65 2004; Padoa-Schioppa \& Assad, 2006; Klein et al., 2008; Lau \& Glimcher, 2008; Xie \& Padoa-

66 Schioppa, 2016). A common currency scale must by definition be amodal, meaning that

67 responses of a value neuron to two offers must be identical if the values of the offers are the

68 same, even if the offers differ in other ways, such as their composition or their location in space.

69 Such abstract value representations are potentially beneficial because they allow for neutral

70 comparison of qualitatively different goods. Consequently, they are central to several models of

71 choice (e.g. Glimcher et al., 2005; Rustichini \& Padoa-Schioppa, 2015).

The orbitofrontal cortex (OFC) has been central to debates about common currency

73 encoding (Tremblay \& Schultz, 1999; Rolls, 2000; O’Doherty et. al, 2001; Wallis, 2007; Padoa-

74 Schioppa, 2011; Padoa-Schioppa \& Schoenbaum, 2015; Wang \& Hayden, 2017). While much

75 work supports the idea that the OFC carries a common currency value representation, a

76 complementary body of literature links OFC to a different and more general function - predicting

77 outcomes (Schoenbaum et al., 1998; Schoenbaum et al., 2003; Kahnt et al., 2010; Schoenbaum et

78 al., 2011; Takahashi et al., 2011; Farovik et al., 2015; Lucantonio et al., 2015). This expectancy 
79 view predicts that neuronal responses should be specific to the properties of the outcome, and not

80 just to its value, so two qualitatively different offers with the same value will generally elicit

81 distinct and unrelated neural responses. These ideas in turn motivated and served as the

82 foundation for cognitive mapping theories, which hold that the brain contains specialized regions

83 whose responses map stimuli to their expected outcomes, and, overall, implement a cognitive

84 map of task space (Wilson et al., 2014; Schuck et al., 2016; Wickenheiser \& Schoenbaum, 2016;

85 Behrens et al., 2018; Schuck \& Niv, 2019).

A good deal of evidence supports the idea that the brain contains neurons whose

87 responses reflect value. In particular, several brain regions include neurons with statistically

88 equivalent responses to equally-valued offers that are defined by different combinations of the

89 same attributes (Padoa-Schioppa \& Assad, 2006; Rudebeck \& Murray, 2014; Strait et al., 2014;

90 Rudebeck et al., 2017; Azab \& Hayden 2020). For example, neurons in the orbitofrontal cortex

91 (OFC, area 13) will respond the same way to two offers with, respectively, a small amount of a

92 more preferred juice and a larger amount of a less preferred juice (Padoa-Schioppa \& Assad,

93 2006). Likewise, neurons in the ventromedial prefrontal cortex (vmPFC, area 14) show a positive

94 correlation between regression weights for the stakes and probability of risky offers (Strait et al.,

95 2014). Both results reflect the same underlying process - a shedding of information (i.e.

96 abstraction) about the details of the factors that produce the value. However, these findings are

97 fundamentally negative ones - they identify a small range of conditions in which neurons fail to

98 distinguish equally valued but different offers, leaving open the possibility that common

99 currency coding is violated in the more general case of dissimilar goods. 
102 So \& Stupohorn, 2010; Kim et al., 2012; So \& Stuphorn, 2016; Farashahi et al., 2019). Decision-

103 makers, both in the lab and in the world, are often faced with a choice between options that offer

104 either a guaranteed sum (e.g., \$10) or the result of an unpredictable stochastic process (e.g., a

$10550 \%$ chance of $\$ 0$ and a 50\% chance of $\$ 20)$. Notably, risky and safe options are psychologically

106 different in several respects - for example, the risky option may elicit a greater potential for

107 learning or engender a different affective response (Lopes, 1987; Loewenstein et al., 2001;

108 Barseghyan et al., 2013). Nonetheless, humans and monkeys can adroitly compare risky and safe

109 offers to each other in an economically meaningful way (Heilbronner, 2017). Consequently,

110 common currency models necessarily predict the existence of neurons with identical neural

111 responses to equally valued risky and safe offers. Any observable difference between responses

112 to these offers would make them distinguishable and thus violate the core definition of common

113 currency value encoding (Levy \& Glimcher, 2012).

114 Here we asked whether neural responses to risky and safe offers use a common currency

115 value code in any of six core reward regions of the brain. Because of ongoing unresolved debates

116 about the potential locus of abstract value encoding, we investigated six regions using the same

117 task: the ventromedial prefrontal cortex (vmPFC, area 14), OFC, rostral OFC (rOFC, area 11),

118 pregenual anterior cingulate cortex (pgACC, area 32), posterior cingulate cortex (PCC, area 29),

119 and ventral striatum (VS). We used a previously developed risky choice task with asynchronous

120 presentation of offers (Strait et al., 2014). By presenting offers asynchronously, we were able to

121 characterize neural responses to a single offer in the absence of comparison signals, in the

122 absence of fluctuating attention, which may lead to rapid shifts between the encoded offer

123 (Krajbich et al., 2010; Rich \& Wallis, 2016; McGinty et al., 2016; Xie et al., 2018). In all six

124 areas, we found that responses evoked by safe offers were entirely unrelated to responses evoked 
125 by equally valued risky ones. Nor did there exist any subpopulation of neurons whose responses

126 showed similar responses to safe and matched value risky offers. In all cases, a simple classifier

127 readily distinguished risky from equally valued safe offers, even in a subpopulation chosen to

128 have minimal firing rate differences. Finally, risky and safe offers occupied dissimilar population

129 subspaces, meaning that risk and safe responses are not just different, they reflect different

130 population organizational frameworks. These results indicate that a basic criterion of abstract

131 value encoding - common responses to equally valued qualitatively different offer types - is

132 violated in all six putative core value regions. The consistency of these results across regions

133 raises the possibility that the brain does not carry abstract value codes anywhere. They therefore

134 support expectancy-based encoding accounts and provide evidence in favor of economic models

135 that eschew abstract value representations (Hayden and Niv, 2021). 


\section{RESULTS}

\section{Behavior}

We used the risky choice task (Strait et al., 2014; see Methods). On each trial, subjects

139 chose between two offers that varied in magnitude and probability (Figure 1A). Safe offers

140 (12.5\% of offers) provided a small volume of juice $(125 \mu \mathrm{L})$ with $100 \%$ certainty. Risky offers

141 provided either a medium ( $165 \mu \mathrm{L}, 43.75 \%$ of offers) or large ( $240 \mu \mathrm{L}, 43.75 \%$ of offers)

142 volume of juice with a defined probability. The offer types for the two offers were selected

143 independently; for risky offers, the win probability was determined randomly from a continuum

144 of values and indicated unambiguously (0-100\%, $1 \%$ increments). We collected data from six

145 subjects (Macaca mulatta) across a total of 315 sessions comprising 211,884 trials (average

$146 \quad 672.6$ trials per session).

Subjects consistently performed at a high level, were modestly risk-seeking, and did not

148 differ from each other qualitatively (Figure 2A). Details of typical behavior in this task are given

149 elsewhere (in greatest detail in Hayden et al., 2010; and in Farashahi et al., 2018 and 2019).

150 Results of these analyses are not repeated here, except to note that subjects' behavior is quite

151 stable and consistent both within and across sessions (Figure 2C-D), and across subjects in this

152 task. Indeed, all tested patterns closely recapitulate those we have observed using this task in the

153 past (ibid.).

The focus of the present study is on comparing responses to safe and risky offers with

155 equivalent subjective values. To identify the relative values of safe offers, we computed the

156 risky-safe indifference point (Hayden et al., 2010). Separately for each subject and separately for

157 medium and large stakes offers, we calculated the likelihood that the subject would choose the

158 safe offer as a function of the probability of the risky offer. We fit the resulting data with a 
159 sigmoid curve and calculated the point at which the best-fitting curve crossed the indifference

160 line (Figure 2, see Methods). We called the value of the risky option that was equivalent to the

161 value of the safe option the indifference point, and assumed that these options were valued

162 equally.

163 Across all six subjects, the average indifference point for medium magnitude risky offers

164 corresponded to an offer probability of $0.33+/-0.05$ (standard deviation). A risk-neutral subject

165 would have had an indifference point at 0.76 ; the fact that the observed value is lower than the

166 optimal indicates that subjects were risk-seeking (Heilbronner \& Hayden, 2013; Heilbronner,

167 2017). Across all six subjects, the average indifference point for high magnitude risky offers

168 corresponded to an offer probability of $0.11+/-0.04$. A risk-neutral subject would have an

169 indifference point of 0.52). This observation is also consistent with risk-seeking.

170 As we have observed many times in the past, but never previously published, preferences

171 were strikingly consistent across many contexts. For example, indifference points are similar for

172 the first and second offers (offer 1: medium: 0.34; high: 0.11 ; offer $2:$ medium: 0.22 ; high $=$

173 0.11), for offers made early and late in the session (early: medium: 0.29; high: 0.09; late:

174 medium: 0.31; high: 0.13), and when risky offers are appear on the left or right (left: medium:

175 0.27, high: 0.11; right: medium: 0.36, high: 0.12). Data for an example subject are shown in

\section{Figure 2B-D.}


bioRxiv preprint doi: https://doi org/10.1101/2021.01.07.425153; this version posted January 8, 2021. The copyright holder for this preprint (which was not certified by peer review) is the author/funder, who has granted bioRxiv a license to display the preprint in perpetuity. It is made available under aCC-BY-NC-ND 4.0 International license.

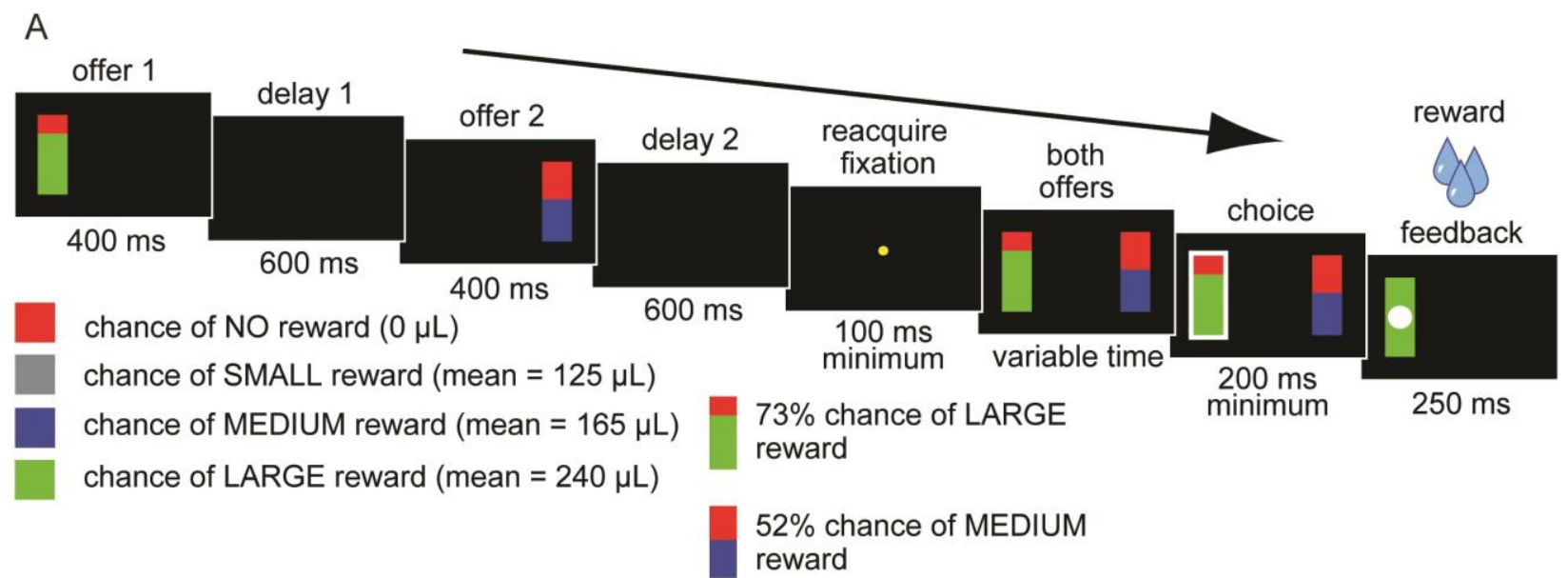

B

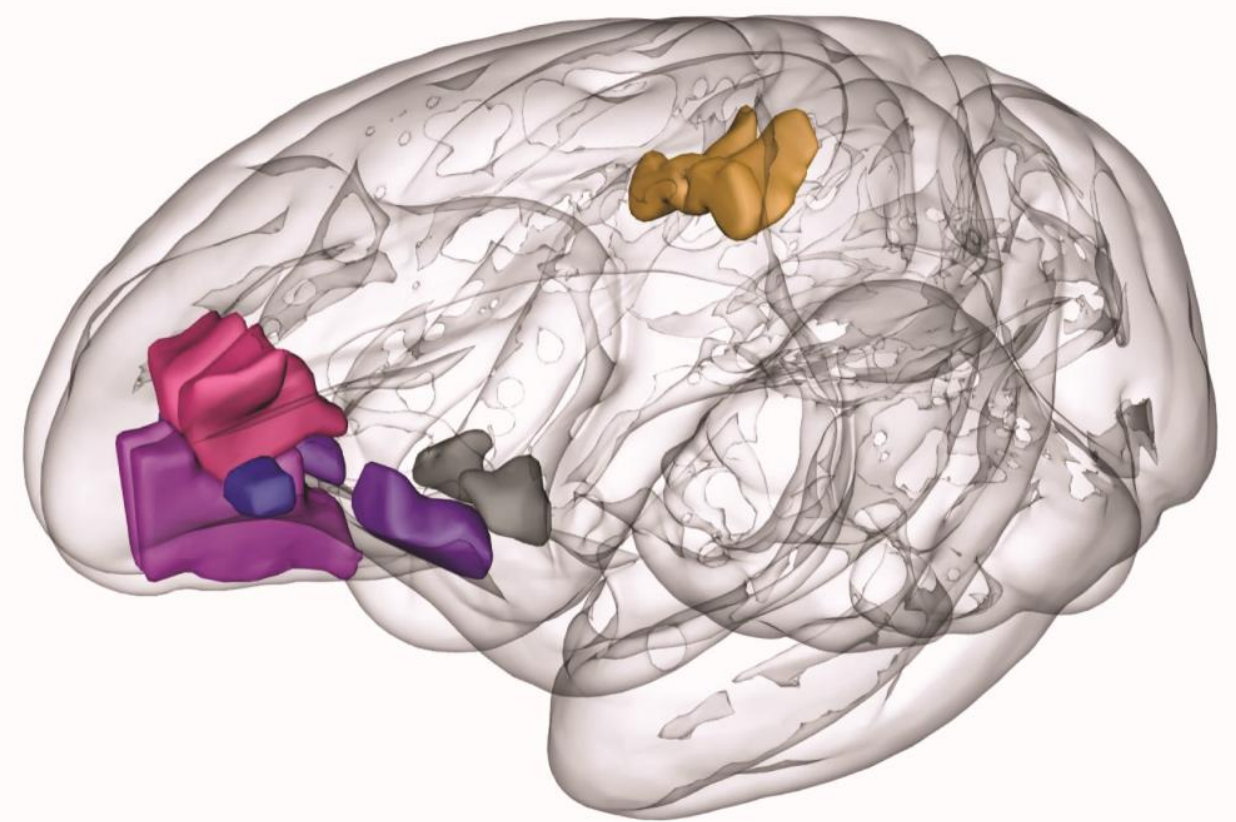

Figure 1. Task, Behavior, and Targeted Structures. (A) Structure of our risky choice task (Strait et al., 2014). Each trial begins with a $400 \mathrm{~ms}$ presentation of the first offer followed by a $600 \mathrm{~ms}$ blank period. Following a $400 \mathrm{~ms}$ presentation of the second offer and another $600 \mathrm{~ms}$ blank period, a fixation spot appears and, on fixation, both offers appear and the subject selects one by saccade. For each offer, the magnitude of the associated reward (stakes) is indicated by the bottom color (green, high or blue, medium) of the stimulus. The probability of being rewarded is indicated by the size of the green/blue segment. (B) Anatomical positions of our brain regions of interest: rOFC (blue), OFC (purple), vmPFC (purple-pink), pgACC (pink), PCC (gold), and VS (grey). 

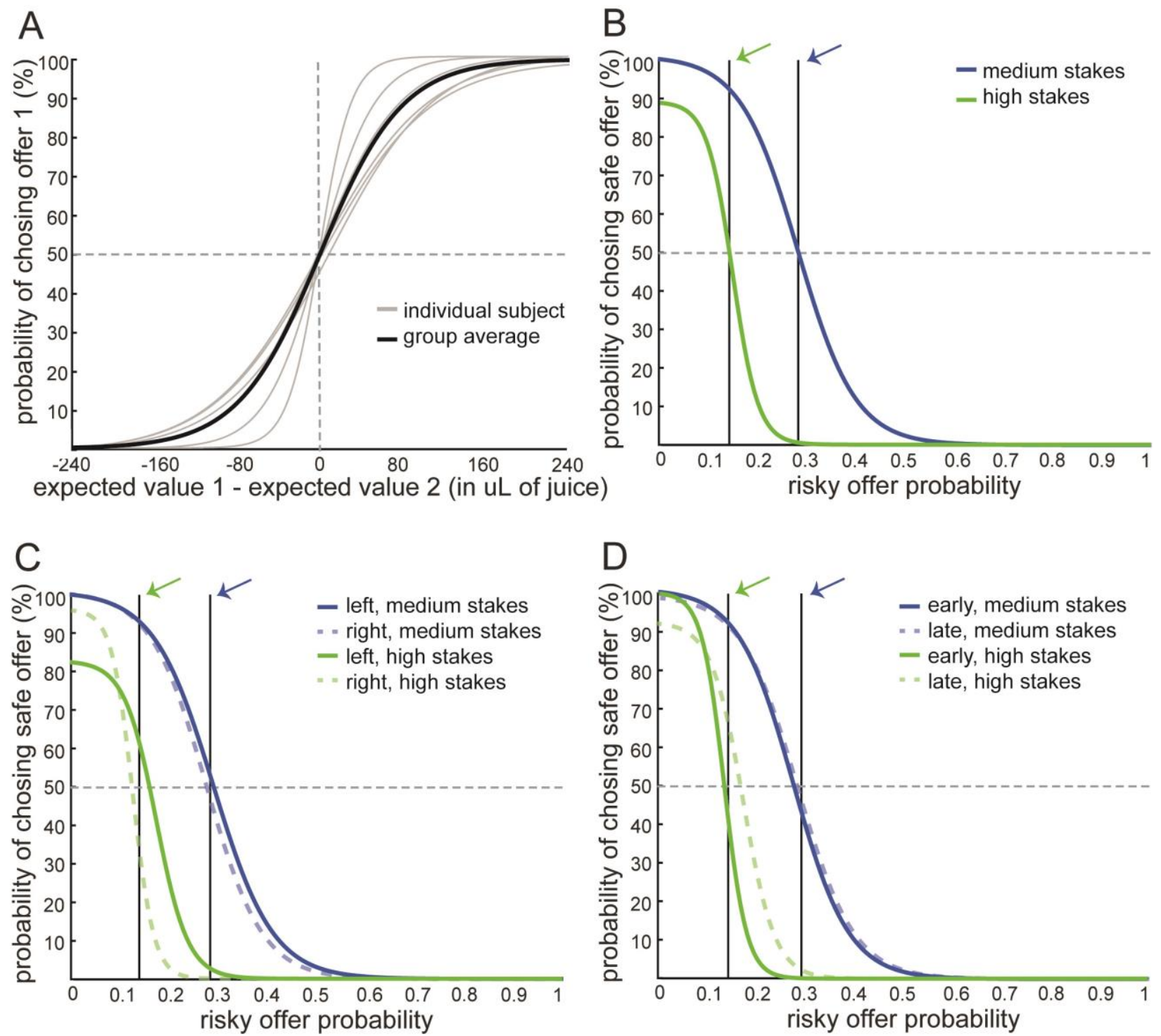

Figure 2. Calculation of equivalent risky and safe values. (A) Likelihood of choosing the first offer as a function of its value relative to the second (specifically, for signed value difference). Sigmoid fits of raw binary data shown (see Methods). Gray lines: individual subjects; black line: group average. In this and subsequent panels, a horizontal, dashed line indicates the indifference point (the point at which choices are 50/50). (B) Likelihood of choosing a safe option as a function of the probability of the risky option for medium (blue) and high (green) stakes offers. All data were analyzed on a subject-by-subject basis, so only data for one example subject (subject B) are shown. Other students showed similar patterns. Vertical black lines (B-D) indicate the probability used as the SV-equivalence point for the subject (the arrow points to the indifference point for medium (blue) and high magnitude (green) risky offers). (C) Same as B, except data are separated for left and right offers. Side of presentation does not affect choice much. (D) Same as C, except data are separated by trials that were in the first (early) or second (late) half of a session.

Neuronal responses to equally preferred risky and safe offers are unrelated 
the risky choice task: vmPFC (area 14, 156 neurons), OFC (area 13, 157 neurons), rOFC (area

207 (nucleus accumbens, 124 neurons). Regions are illustrated in Figure 1B and anatomical

208 boundaries are provided in the Methods. We recorded in two subjects for all areas, although

209 different subjects were used for the different areas (see Methods). Detailed analyses of responses

210 to risky offers were reported previously for vmPFC and VS (Strait et al., 2014; Strait et al.,

211 2015). We have not previously examined responses to safe offers.

212 We reasoned that any neurons that use a common currency code for offer value must

213 produce identical neural responses to subjectively equivalent (that is, equally preferred) risky and

214 safe offers (Padoa-Schioppa \& Asaad, 2006; Kennerley et al., 2009; Levy \& Glimcher, 2012).

215 Using each individual's subjective indifference point, we then defined a range of probabilities

$216(+/-2.5 \%$, total range of 5.0\%) and treated all offers within that range as being subjectively

217 equivalent to the safe value. Note that we subsequently checked for robustness by repeating the

218 following analyses using a larger range (+/- 5\%, total range of 10\%) but because we found no

219 qualitative differences, we do not report those results.

Our analyses focused on the first offer epoch, a $500 \mathrm{~ms}$ analysis window starting $100 \mathrm{~ms}$

221 after the onset of the first offer. We have used this epoch in all our past research on this and

222 similar tasks (Strait et al., 2014, 2015, and 2016; Azab \& Hayden, 2017, 2018, and 2020). We

223 have found that this epoch provides a good characterization of functional responses and allows

224 for fair comparison across brain regions (Strait et al., 2016; Maisson et al., 2020). We used it

225 here for those reasons and because adherence to a single pre-planned epoch of interest reduces

226 the likelihood of inadvertent "p-hacking".

227 Neuron vmPFC.70 (Figure 3A) showed differing selectivity for safe and risky offers 
228 during the first offer epoch. Neuron pgACC.17 (Figure 3B), recorded from subject B, showed

229 clear and roughly monotonic tunic for probability of medium and high magnitude risky offers.

230 This means that a downstream decoder could, in principle, readily interpret the firing to identify

231 the probability (and thus, in the context of this task, the value) of a risky offer. It does so because

232 the response of the neuron reflects a single consistent scale for risky offers. However, this scale

233 does not appear to extend to safe offers. Because the safe offer had a subjective value equivalent

234 to 0.28 , its mean neural response should have been the same as the response to that offer $(0.36$

235 spikes/second) if risky and safe offers used a common scale. Instead, it evoked a mean response

236 of 1.67 spikes/second. (Note that the second quantity is significantly lower than the first,

237 Student's t-test, $\mathrm{t}=4.31, p<0.001)$. In other words, while this pgACC neuron appears to use a

238 consistent code for risky offers, it does not appear to use the same code for both safe and risky

239 offers. Additional sample cells, from each targeted area, showed positive and negative

240 monotonic tuning while having still clearly responded differently to safe and risky offers (Figure

241 3C-F).

242 


\section{A}
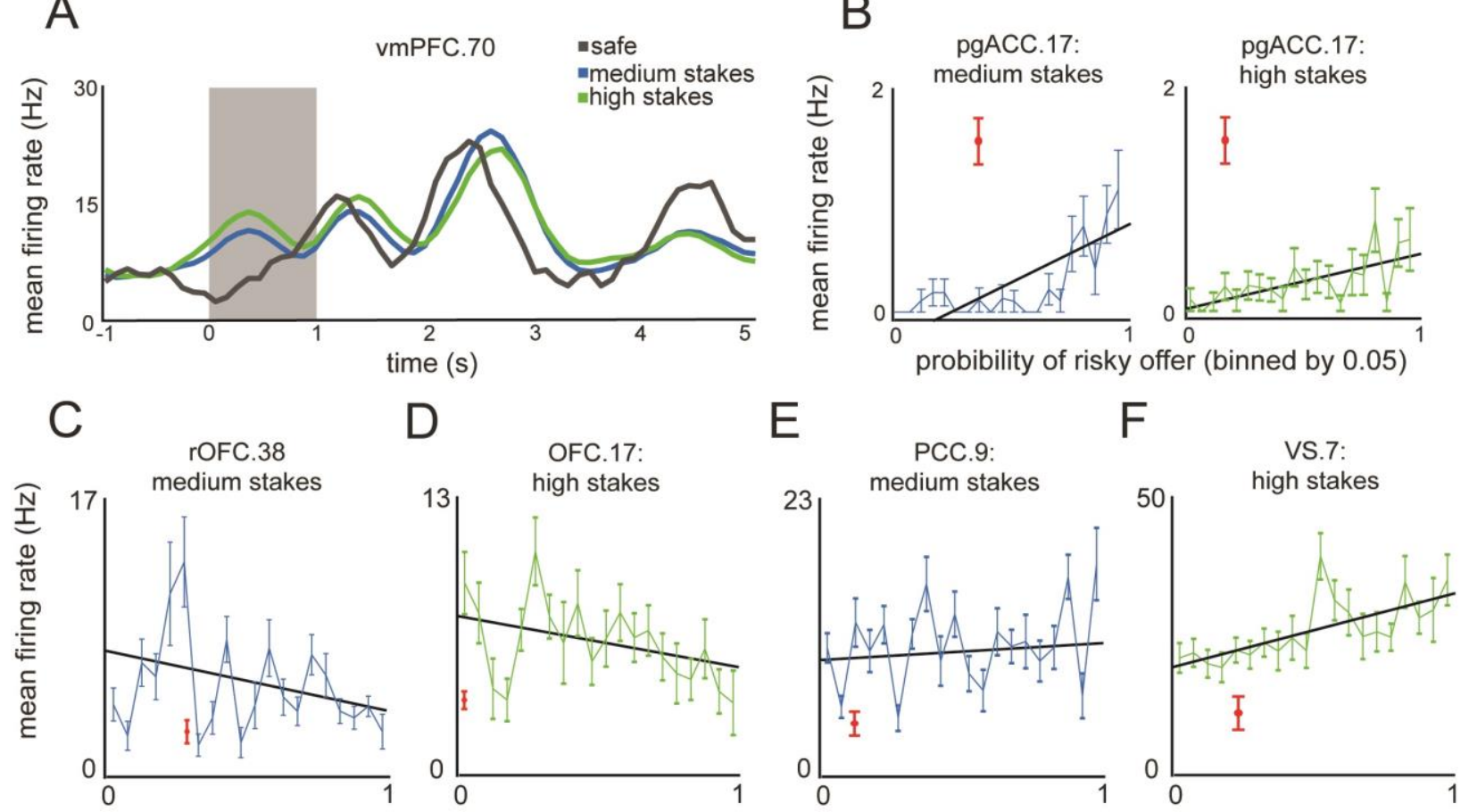

243

244

245

246

247

248

249

250

251

252

253

254

255

256

257

258

259

260

261

262

263

264

265

Figure 3. Responses of single neurons. This figures shows the average responses of sample neurons to safe and risky offers of differing values, as well as the average response similarity rates. (A) Peristimulus time histogram from mean firing rates of sample neuron vmPFC.70. Each line indicated the average response across offers of a given risk profile (grey: all safe offers; blue: all medium magnitude risky offers; green: all large-magnitude risky offers). The grey shaded box indicates the 1-second period from which the 500-ms epoch 1 analysis window was extracted, where the onset of the first offer is time-locked to zero seconds. (B) This is a plot of data collected from a sample neuron in the OFC, which showed a response to safe offers that was statistically different from the response to equivalent risky offers. Depicted are the average responses to medium magnitude (left; blue) and high (right; green) stakes, separated by probability ranges of 0.05 . The red point indicates the average response of the given neuron to safe offers (error bars denote the SEM across responses to safe offers). The diagonal black line indicates a fitted regression line, showing positive monotonic tuning. (C-F) Same as (B), but demonstrating sample cell responses to an assortment of medium and high stakes offers from across all target areas.

\section{Responses to safe offers are unrelated to responses to equally valued risky offers}

To test whether risky and safe offers are encoded in similar ways in any of our six brain areas, we focused on the key variable, the difference in firing rate response evoked by the two offer types. We call this quantity the evoked response difference, or the delta for short. If a neuron uses a common currency code for value, then the delta must necessarily be zero. In practice, delta will inevitably deviate from zero due to measurement noise. Specifically, because 
neuronal responses are stochastic, responses to two different stimuli will necessarily be measured as different even if the true responses are identical. We accounted for this issue by using an analysis approach that combines positive and negative control analyses.

The goal of the negative control analysis was to ascertain whether deltas for safe and

270 equally valued risky offers are lower than one would expect by chance. We did this by

271 comparing the difference between the safe response and the response to a randomly chosen

272 probability in the range of 0.0-1.0 (by 0.01 units). In other words, this analysis asks, in effect,

273 whether the safe offer has a special relationship (specifically, a smaller delta) with the

274 equivalently valued risky offer that it does not have with any randomly selected risky offer. If it

275 does not, that implies that risky and safe offers are encoded using unrelated codes.

277 an average response of 15.71 spikes/second and equivalent medium value risky offers evoked an

278 average response of 19.85 spikes/second. Its delta was therefore the difference between these 279 two numbers, or 4.14 spikes/second. This value is large - roughly $20 \%$ of the risky response 280 but, how large is this value relative to what would be expected by chance? For this neuron, the 281 average delta generated using randomly chosen probabilities (rather than the equivalently valued 282 one) was $2.73+/-1.16$ spikes/second (this number reflects an average over 1000 randomly 283 sampled probabilities). The observed (true) delta is larger, not smaller, than the control (random) 284 delta, arguing against the hypothesis that this neuron has a common currency code for value.

285 Moreover, these two deltas were not significantly different, $(p=0.68$, bootstrap test, see

286 Methods). In other words, for this neuron, the response evoked by the safe offer is not

287 significantly lower than the value evoked by any random offer - it's within the range of values

288 one would expect by chance. To put it another way, a downstream decoder would have no way 
to preferentially associate the safe offer with its equivalently valued risky offer and the evidence

290 suggests the codes for safe and risky offers are entirely unrelated.

We performed this analysis for all recorded neurons in vmPFC. To account for possible

292 scaling differences between responses of different neurons, we used normalized (Z-scored) -

293 firing rates, although the conclusions were unchanged when using raw firing rates. We found that

294 the average normalized delta across neurons for safe and equally preferred medium magnitude

295 risky offers was $0.068+/-0.007$ (standard deviation) $\mathrm{z}$-score units; the average for the random

296 deltas was similar $(0.068+/-0.002$; these were not different, $p=0.487$, bootstrap test, see

297 Methods). The average normalized delta for safe vs. high magnitude was 0.071; the average for

298 the random deltas was 0.073; these were also not different, $p=0.519$; Figure 4B-C). In other

299 words, neither of these average deltas was significantly smaller than the average deltas between

300 randomly chosen risky offers (using the bootstrapping method; see Methods). Note that because

301 there were two conditions, and thus two possibilities of detecting a common currency code, it is

302 appropriate to correct for multiple comparisons; the Bonferroni corrected $\mathrm{p}$-values are $p=0.698$

303 and $p=0.720$, for medium and high magnitude risky offers, respectively. We found the same

304 patterns in the other five structures we examined - most importantly, no area showed a

305 measurable difference between deltas from safe and random risky offers (summarized in Figure

306 4B-C).

307 The goal of the positive control is to show that our failure to find a difference between

308 the true and random conditions is not itself due to high noise in our sample. In other words, it is

309 theoretically possible that there was enough noise in our measurement of evoked responses that

310 we would have detected a large but spurious delta even if there were no true difference. Our

311 positive control analysis is designed to exclude this possibility by confirming we could indeed 
312 detect a lower than random delta if it existed. (One intuitive way to think about this analysis is

313 that it asks whether we collected sufficiently large numbers of neurons in each area to detect a

314 true common currency code if it existed).

315 To do this, we randomly assigned trials from SV-matched offers to two sets of equal size,

316 for each neuron. We then computed the delta for each neuron between these two randomized

317 sets. Because these responses are evoked by stochastically identical stimuli, they must have zero

318 true difference. Any measured difference between them gives a measure of what difference we

319 would expect to arise by chance. We reasoned that if the high measured values of our deltas

320 reflected a true violation of common currency coding, they must have necessarily been larger

321 than the responses to the randomly sub-selected sets of risky trials.

322 We found that the average delta across random sub-selections, computed from vmPFC

323 responses, was $0.051 \mathrm{z}$-score units. This value was lower than the safe vs. medium magnitude

324 true deltas in vmPFC (which was $0.068, p<0.001$, bootstrap test). This positive control delta

325 was also significantly lower than the safe v. high magnitude true delta $(0.071, p<0.001$,

326 bootstrap test). Similar differences were evident between deltas computed from neural responses

327 in all six structures $(p<0.01$ in all cases, Figure 4C). This analysis indicates that the effects we

328 see are not due to insufficient or noisy data, but instead reflect a true and robust violation of

329 common currency coding. 
A

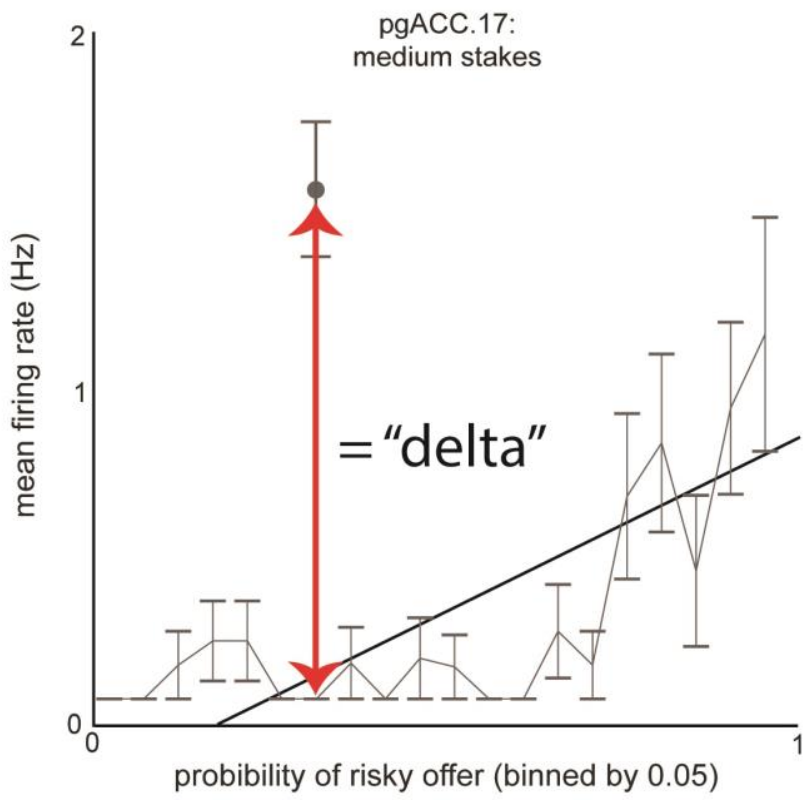

C
B

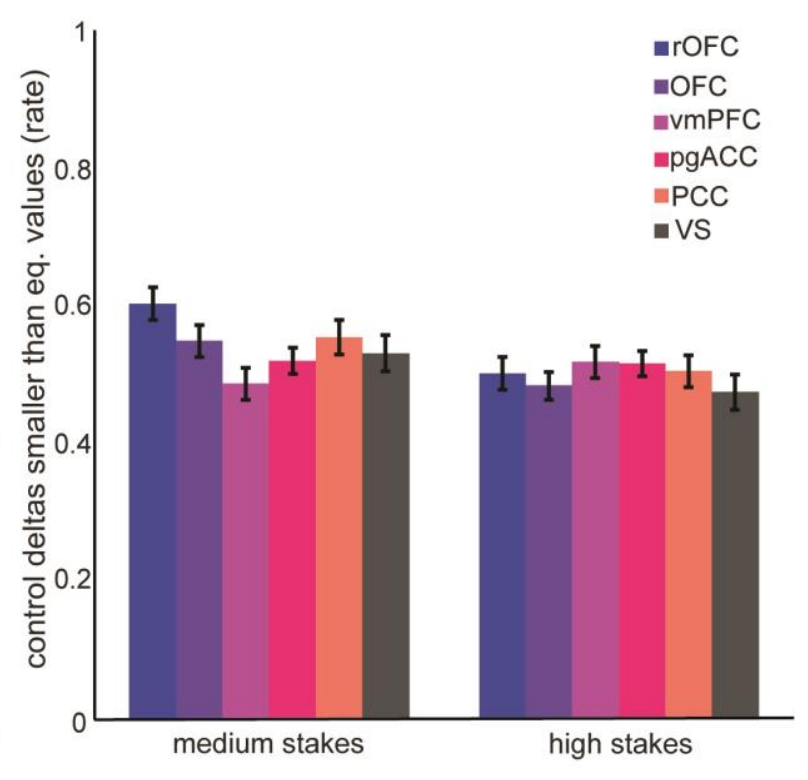

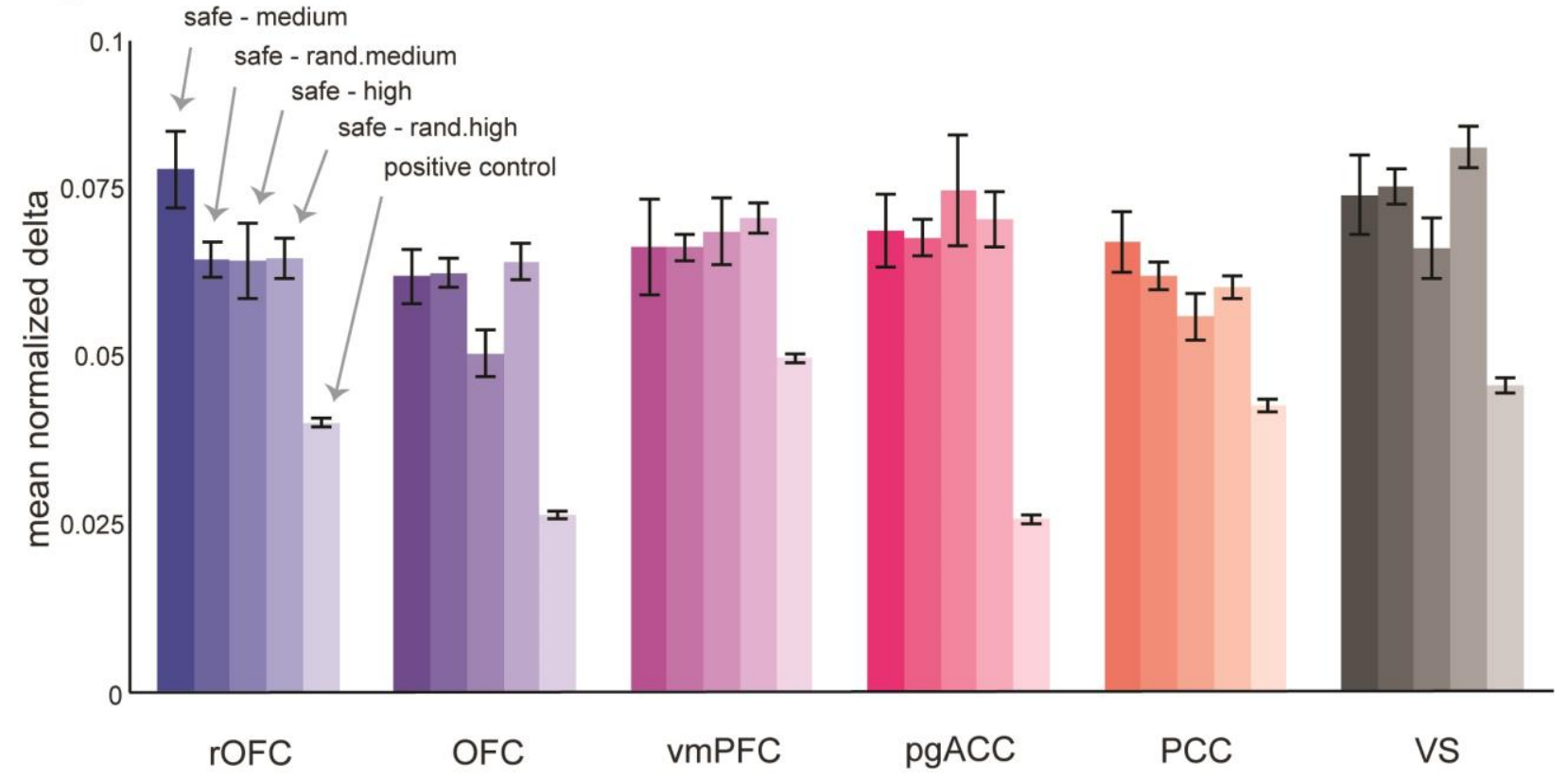

Figure 4. Average risk-safe response difference (delta). (A) The same cell depicted in Figure 3B (left pane). The red arrow links the responses to the safe and SV-matched risky offers. The absolute value of their difference constitutes the delta. (B) The average proportion of deltas, across neurons, between random offers that are smaller than the delta between equivalent safe and risky offers (i.e. the $p$-value from our bootstrap test). The common currency hypothesis would predict that safe - risky deltas are always significantly less than safe - rand.risky deltas. (C) The average normalized evoked response difference (delta) for each structure. From left to right (most to least opaque), for each structure: deltas in response to safe v. medium magnitude risky offers, safe $v$. randomly selected medium offer, safe $v$. high, safe v. random high, and the positive control deltas. The fact that observed deltas are generally greater than or equal to (never significantly less than) the control deltas (safe - rand.risky) indicates a violation of the common currency hypothesis. 


\section{No special subpopulation of abstract value encoding neurons}

In many cases, common currency coding is not a property of all neurons in a region, but

348 only a subset of specialized abstract value cells (often called offer value cells, e.g. Padoa-

349 Schioppa \& Assad, 2006). Our approach, which looks at average response differences in the

350 population, would be sufficient to detect abstract value encoding, even if it were limited to a

351 subpopulation of cells, because those cells would pull down the average delta for the population;

352 our use of the positive control approach (see above) means we can be confident we could detect

353 them even if they were in a very small minority. Thus, that result, while indirect, is still sufficient

354 to cast strong doubt on the idea that there are subpopulations of abstract value cells. Nonetheless,

355 we wanted to more directly test the hypothesis that there is a specialized subpopulation of pure

356 value cells.

We started by reasoning that such a subpopulation, if it exists, will be defined by having

an unusually low delta (i.e. low difference between responses evoked by safe offers and

equivalently valued risky ones). In theory, that delta would be precisely zero, but (as we state

361 necessarily be lower than the delta for the set of non-value neurons. A putative value-coding

362 population, then, can be identified by taking the subset of neurons with the lowest delta value.

364 control analysis. Specifically, we can ask whether, given a presumed subpopulation size, the

365 group average delta associated with this subpopulation differs from the group average delta for a

366 matched dummy set of cells identified by using a random probability. If the value is not lower,

367 that argues against the idea that a specialized subset of pure value neurons of a specific set size 
exists. Because we had no a priori hypotheses about the likely size of such a set, we tested all

369

370

371

372

373

374

375

376

377

378

379

380

381

382

383

384

385

386

387

388

389

390 possible set sizes.

Consider, for example, the possibility that $30 \%$ of neurons in OFC $(n=47 / 157)$ may constitute a unique subpopulation of cells whose responses encode value abstractly. These neurons could be identified by finding those $30 \%$ of cells with the lowest difference between responses to safe and equivalent valued risky options (that is, lowest deltas). We then identified these neurons - their average delta was found to be $0.012 \mathrm{z}$-scored units. Next, we picked a random probability (say, 0.65 ) and identified another set of $47 / 157$ neurons whose deltas for that probability are minimized. The average random delta for this set of neurons was found to be 0.017 (we can call this the pseudo-delta for 0.65). We next repeated this process 1000 times with 1000 random probabilities, and averaged the resulting pseudo-deltas. The average of these turned out to be 0.015 (Figure 5A). Then we asked whether the delta for the equivalent probability was lower than the average pseudo-delta for the random probability (as we would expect if this subpopulation has, or even approximates, an abstract value code). They were not $-p=0.171$.

This result, then, indicates that there is no unique subpopulation of size $30 \%$ in OFC.

We next repeated this process for all possible population sizes $(1-100 \%$, by $1 \%$

increments). (Note that the $100 \%$ condition in this test is mathematically equivalent to what we call the negative control delta analysis in the previous section, so this analysis serves as a generalization of that one to all possible set sizes smaller than $100 \%$ ). As the size of the population increased, naturally so did the average delta across the subpopulation because we necessarily selected more neurons with larger individual deltas (Figure 5B). The random deltas also must show the same pattern of increase. The important comparison is to determine whether the safe-risky deltas and safe-random pseudo-deltas, across subsampled population sizes, differ 
391 from each other statistically. For each subsampled population size, we computed the deltas both

392 between equivalent and between random offers and performed a Komologorov-Smirnov test,

393 comparing the pair of equivalent and random delta vectors across subset size. We found that

394 there were no significant differences between the equivalent and control deltas as a function of

395 subset size in the other targeted structures ( $p>0.05$ in all cases; Figure 5C-H).
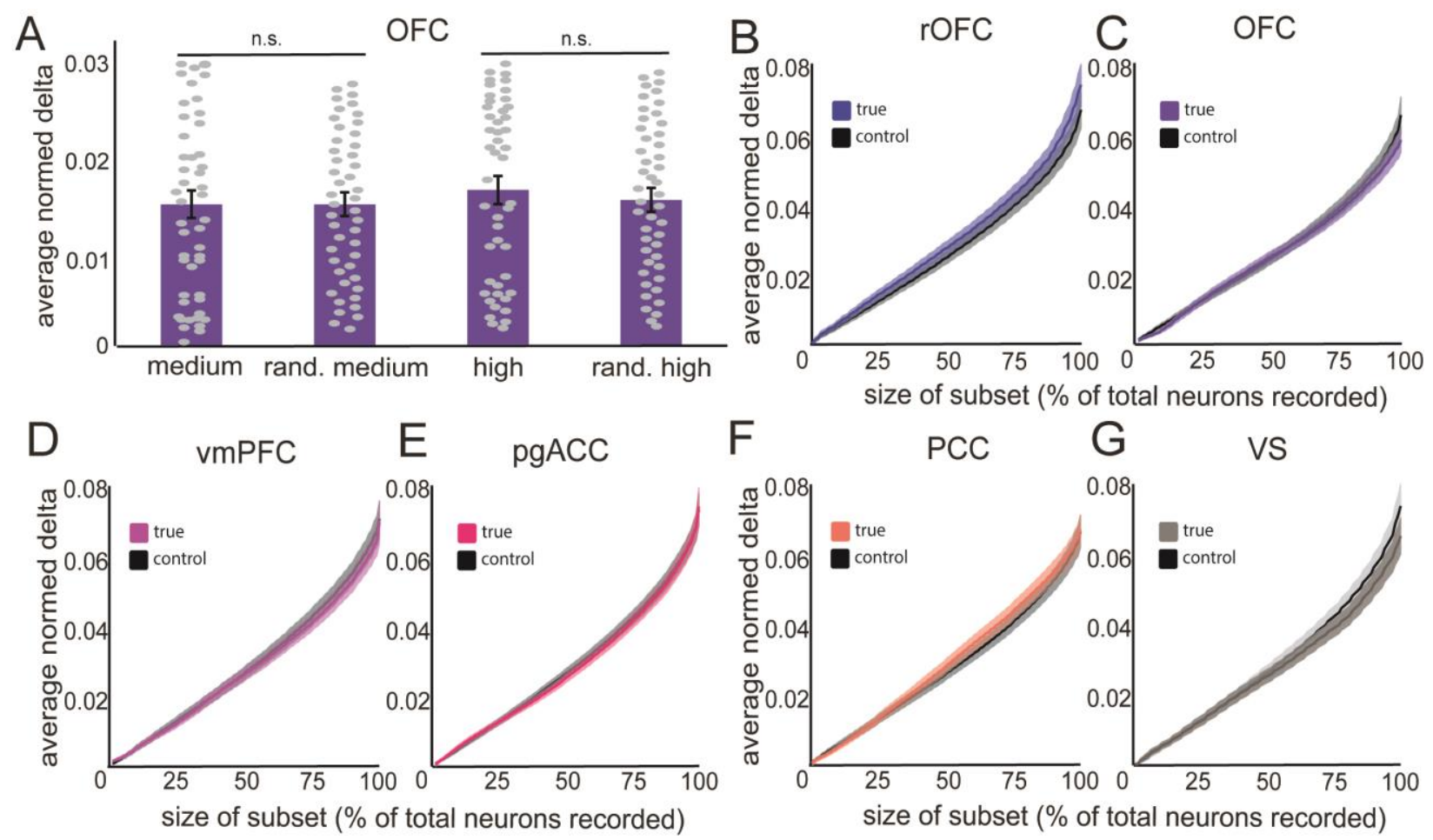

$\mathrm{H}$

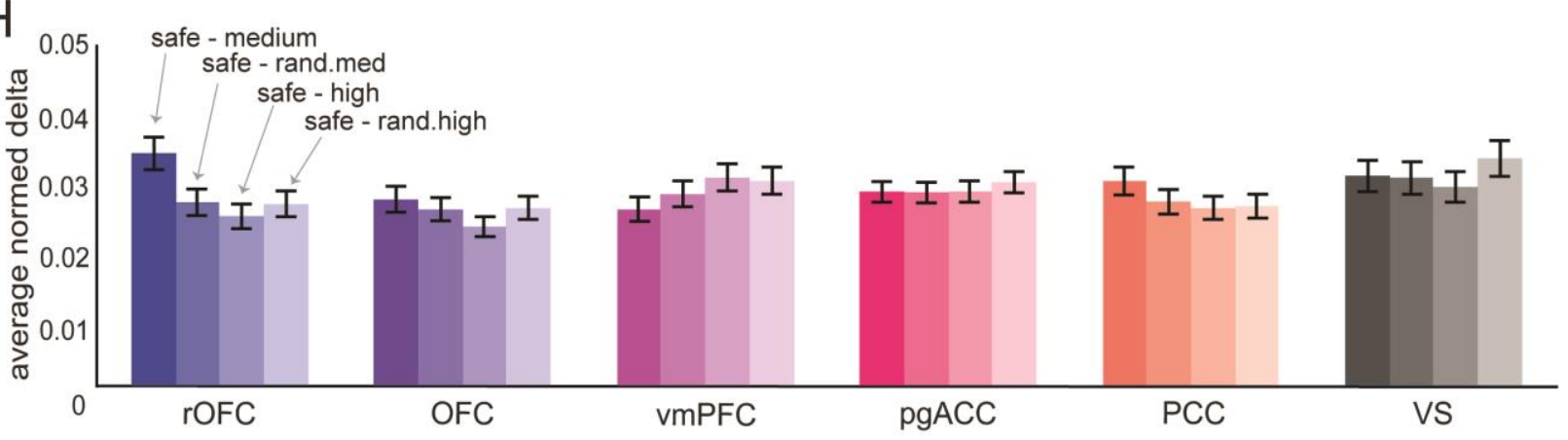

Figure 5. Delta Analysis with best subsets of various sizes. This figure depicts our analysis of response differences in best subsets of neurons. (A) For an example structure, OFC, we show the comparison between deltas at example subset size of $30 \%$ of recorded OFC neurons. Bars indicate the average normalized deltas for a given comparison: safe v. medium stakes, random medium stakes offers, safe $v$. high stakes, random high stakes offers. Each dot indicates the delta for a single neuron. Error bars indicate the standard error across the subset. (B) Shown is the change in average delta (for visualization 404 only: collapsed across both medium and high stakes comparisons) between safe-risky (blue) and safe- 
random (black). The lines indicate the average normalized deltas (as in panel A), across subsets from a size of $1-100 \%$ of recorded neurons. Shaded ribbons denote the standard error across the subset. *Note again that collapsing across medium and high stakes offers is for visualization only. (C-G) same as (C), but for each area of interest. The analysis was performed for safe-medium and safe-high independently. (H) For each structure, each bar provides a summary of the average normalized delta across all subset sizes. Error bars represent the standard error across subset sizes.

\section{Decodability of safe and equivalently valued risky offers}

If the brain specifically encodes value in a common currency manner, then it should not be possible, in principle, to decode safe from risky offers. If, conversely, the brain uses distinct codes for the two categories, then the category (risky vs. safe) should be readily decodable even if the offers are equally valued. We used a standard classifier approach to ask this question. Specifically, we trained a binary support vector machine (SVM) to decode safe from equally valued risky offers based on neural responses (see Methods). We then cross-validated the trained model by using it to predict risky vs. safe from responses in a sequestered set.

In OFC, for example, we found that the classifier could readily disambiguate safe from equally valued risky trials (medium magnitude: $\mathrm{t}=730.1, p<0.001$; high magnitude: $\mathrm{t}=780.5$, $p<0.001)$. This difference is quite large. For comparison, a t-test comparing $50 \%$ to $0 \%$ average accuracy along cross-validations, given our pseudo-population method (see Methods), and assuming equal variance, would be about the same $(\mathrm{t}=701.7)$. We also observed clear decodability in the other five structures $(p<0.001$ in all cases; Figure 6A).

Next, we wanted to confirm that the high decodability rate was not an artifact of using all recorded neurons, including those with stark differences in their responses to equally valued safe and risky offers. We reasoned - as we did above - that there could still be a special subpopulation of pure value cells, such that their abstract encoding of value was obscured by the other neurons. Thus, we identified putative pure-value cells as the set of cells with no significant difference in response to safe and equivalent risky offers (that is, $p>0.05$ ). Then, to be even more 
conservative, we included only the half of these cells with the smallest difference between

434 responses to safe and SV-matched risky offers. In the OFC, this constituted 68/157 neurons

435 (43.3\% of recorded cells; rOFC: 60/138 neurons, 43.5\%; vmPFC: 66/156 neurons, 42.3\%;

436 pgACC: 100/255, 39.2\%; PCC: 60/151, 39.7\%; VS: 50/124, 40.3\%). As before, in OFC we

437 found that the classifier could readily disambiguate the safe from the SV-matched risky trials

438 (medium magnitude: $\mathrm{t}=428.5, p<0.001$; high magnitude: $\mathrm{t}=513.2, p<0.001$ ). This pattern

439 was observed in all five of our structures $(p<0.001$; Figure 6B).

To push even harder against our own conclusions, we conducted the same analysis on an

441 even smaller subset. We included only a quarter of the cells with the smallest difference between

442 responses to safe and SV-matched risky offers. That is, we took the subpopulation that would, by

443 even a very conservative analysis, be most likely to be classified as pure value cells. In the OFC,

444 this constituted 39/157 neurons (24.8\% of recorded cells; rOFC: 30/138 neurons, $21.7 \%$;

445 vmPFC: 33/156 neurons, 21.2\%; pgACC: 50/255, 19.6\%; PCC: 30/151, 19.9\%; VS: 25/124,

$44620.2 \%$ ). Even so, in OFC, we found that the classifier could readily disambiguate the safe from

447 the SV-matched risky trials (medium magnitude: $\mathrm{t}=416.2, p<0.001$; high magnitude: $\mathrm{t}=391.4$,

$448 \quad p<0.001)$. Again, this pattern was still observed in all five of our structures $(p<0.001$, in all

449 cases; Figure 6C).

A

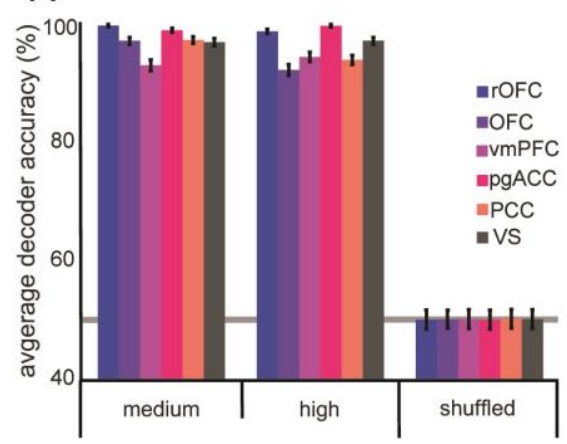

B

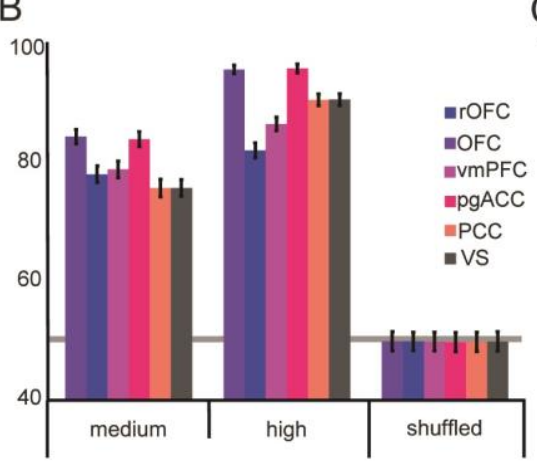

C

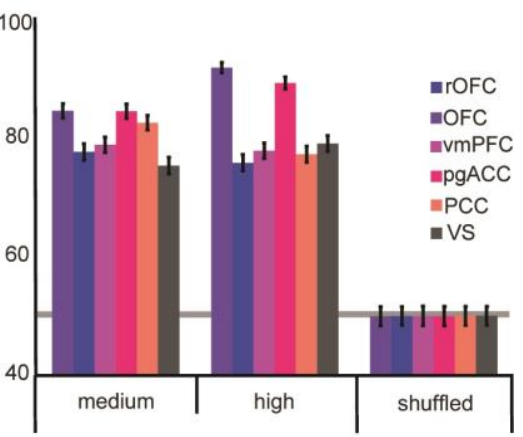

Figure 6. Safe and equivalently valued risky offers are readily decoded. (A) Decodability for safe and risky offers is high in all areas for both medium and high stakes gambles. Shuffled data refers to 
454

455

456

457

458

459

460

461

462

463

464

465

466

467

468

469

470

471

472

473

474

475

476

477

478

479

480

481

decodability of randomly assigned safe/risky labels to neural responses that are completely shuffled across trials and cells. Error bars indicate the standard error across cross-validations. (B) same as A, except that the only neurons used in the decoder were those which, in the first analysis, showed no significant differences in neural responses to safe and equivalent risky offers, and which have differences in responses within the lowest half of the set. (C) same as B, except that the only neurons used are those with differences in responses within the lowest quarter of the set

\section{Response subspaces for safe and risky offers are different}

Responses of ensembles of neurons have correlated variability; these correlations restrict ensemble responses to specific subspaces (Oşan et al., 2007; Gallego et al., 2017). Emerging evidence indicates that neuronal populations can move between subspaces and that subspace reorganization can serve a partitioning function, for example, between motor preparation and execution (Elsayed et al., 2016) or between evaluation and comparison (Yoo \& Hayden, 2020).

The common currency hypothesis would require the use of a common abstract value subspace for different offer types with the same value. Here we asked whether each of our six regions make use of common or distinct subspaces for encoding risky and safe offers.

To do this, we followed an approach to characterize the uniqueness of subspaces for temporally distinct offer epochs, and that is based on methods devised to study subspace

reorganization in the motor system (Elsayed et al., 2016; Yoo \& Hayen, 2020). We modified that approach to allow for a comparison between safe and risky subspaces within the same epoch.

Specifically, we performed a principal component analysis (PCA) on neural responses to both risky and safe offers of equal value. For each subject independently, we projected responses evoked by both safe and equivalent risky offers into the safe offer subspace and computed the total percent variance explained by the top ten principal components. We then averaged the explained variances across subjects (Figure 7A-B). We used these projections to quantify the extent to which subspaces were aligned ( $\mathrm{A}_{\mathrm{idx}}$; see Methods, Elsayed et al., 2016). This number quantifies the extent of the variance explained in the safe responses, by projecting them into 
risky response subspace, as a proportion of the variance explained in the safe responses. An alignment index equal to 1.0 would indicate that the variances in risky and safe responses are

484 explained equally well by the principal components that constitute the safe response subspace.

485 That value would suggest common ("aligned") subspaces and is predicted by the common currency hypothesis. An alignment index equal to zero would indicate that the risky and safe

487 subspaces are strictly orthogonal and an index between 0 and 1 would indicate a partial 488 orthogonality; even partial orthogonality would indicate distinct organizational principles, in 489 violation of the common currency hypotheses.

490 We found that OFC safe and medium magnitude risky response subspaces had an 491 alignment index of $A_{i d x}=0.21$. Safe and high magnitude subspaces had an alignment of $A_{i d x}=$

$492 \quad 0.25$. The critical question is whether this value is significantly less than 1.0 , which would 493 indicate at least partial orthogonalization. As a control, to determine the significance of our

494 results, we reasoned that breaking the structure of the within-time covariance, while maintaining 495 the within-neuron covariance, should produce subsets that are more orthogonal than would be 496 expected by the within-neuron covariance structure alone. That is, any alignment index at or

497 below this threshold would be considered primarily orthogonal. To do this, we shuffled the data

498 from across all three response matrices (safe, matched-medium, and matched-large) and

499 computed the alignment index between shuffled sets (see Methods). We repeated this process

500 over 1000 iterations. Then, to test for significance, we computed the $95 \%$ confidence interval

501 across iterations; a value outside of this range can therefore be said to be significant at $\mathrm{p}<0.025$

502 (two-tailed t-test). We found that the average shuffled alignment index in OFC was $\mathrm{A}_{\mathrm{idx}}=0.276$.

503 Both the safe-medium and safe-high alignment indexes were below the $95 \%$ confidence interval

504 (0.274 - 0.278). In other words, response subspaces for safe and equally valued risky offers in 
505 OFC are more orthogonal than we would expect by chance given the inherent statistical

506 properties of our dataset (Figure 7C). We found similar results in all structures (safe-medium

507 and safe-high was below the $95 \%$ confidence interval, in all cases; $\mathrm{p}<0.025)$.

508 Our results suggest that the subspaces are generally more orthogonal than they are

509 aligned. However, we wanted to confirm that regardless of the degree of alignment, the safe,

510 medium, and high stakes neural responses are readily separable when projected into the safe

511 offer subspace. To do this, we again projected each of the neural response matrices into the safe

512 offer subspace (Figure 7D). We then performed a multinomial logistic regression (to

513 discriminate class based on linear trends within the projections), using the projections onto the

514 top 10 principal components to predict whether the offer was safe, medium, or high stakes. We

515 found that, in all structures, the regression model was significantly able to discriminate between

516 offer types (rOFC: $\mathrm{F}=13.36, p<0.001 ;$ OFC: $\mathrm{F}=13.33, p<0.001 ;$ vmPFC: $\mathrm{F}=13.32, p<$

517 0.001; pgACC: $\mathrm{F}=13.26, p<0.001 ; \mathrm{PCC}: \mathrm{F}=13.26, p<0.001 ; \mathrm{VS}: \mathrm{F}=13.29, p<0.001)$. Since

518 the factors used in the regression model are all projections into safe subspace, these results

519 suggest that safe and risky offers operate in highly separable hyperplanes. That is, safe and risky

520 information can likely be easily demixed in a given subspace, in striking violation of the

521 common currency hypothesis. 

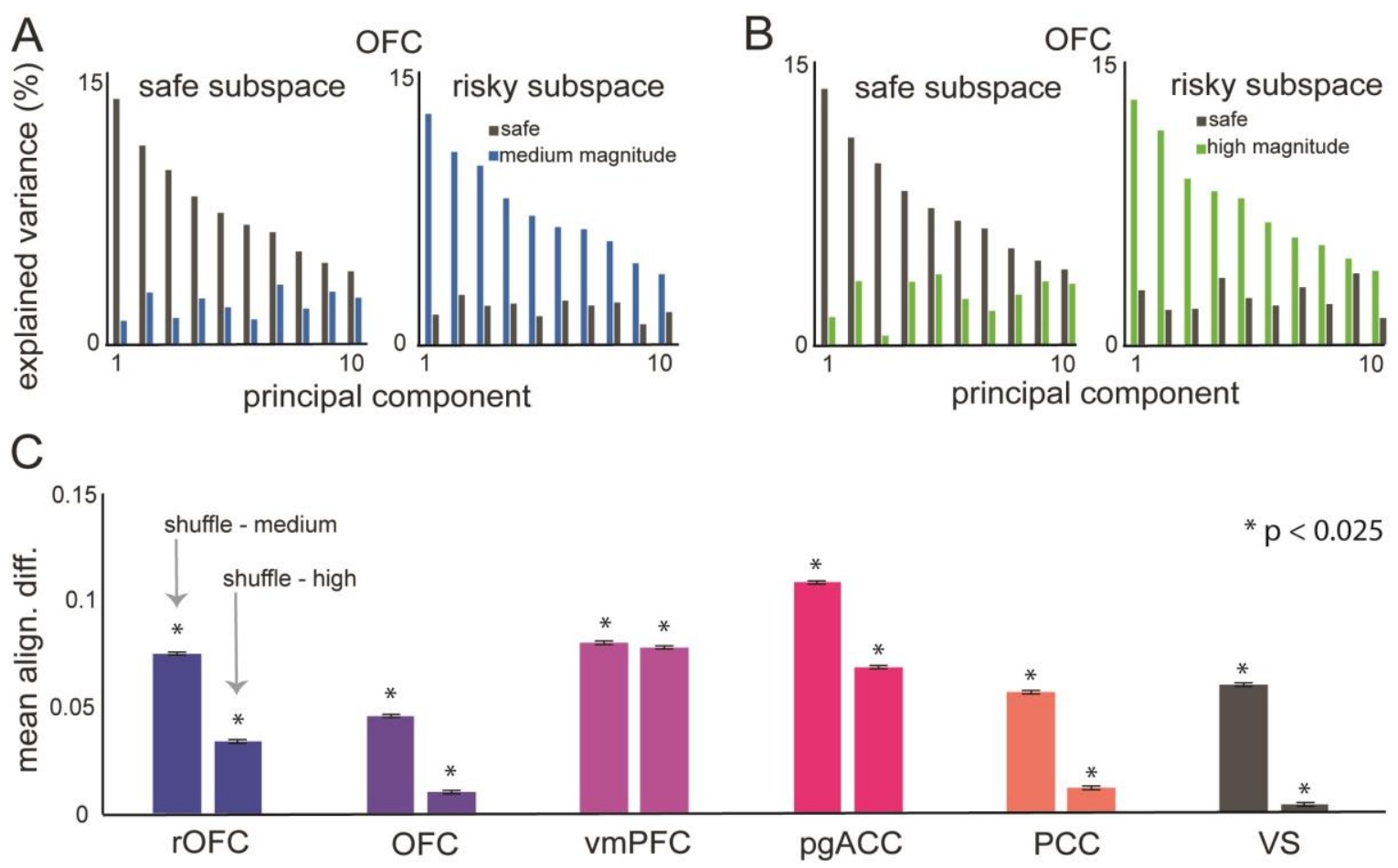

D vmPFC: safe subspace
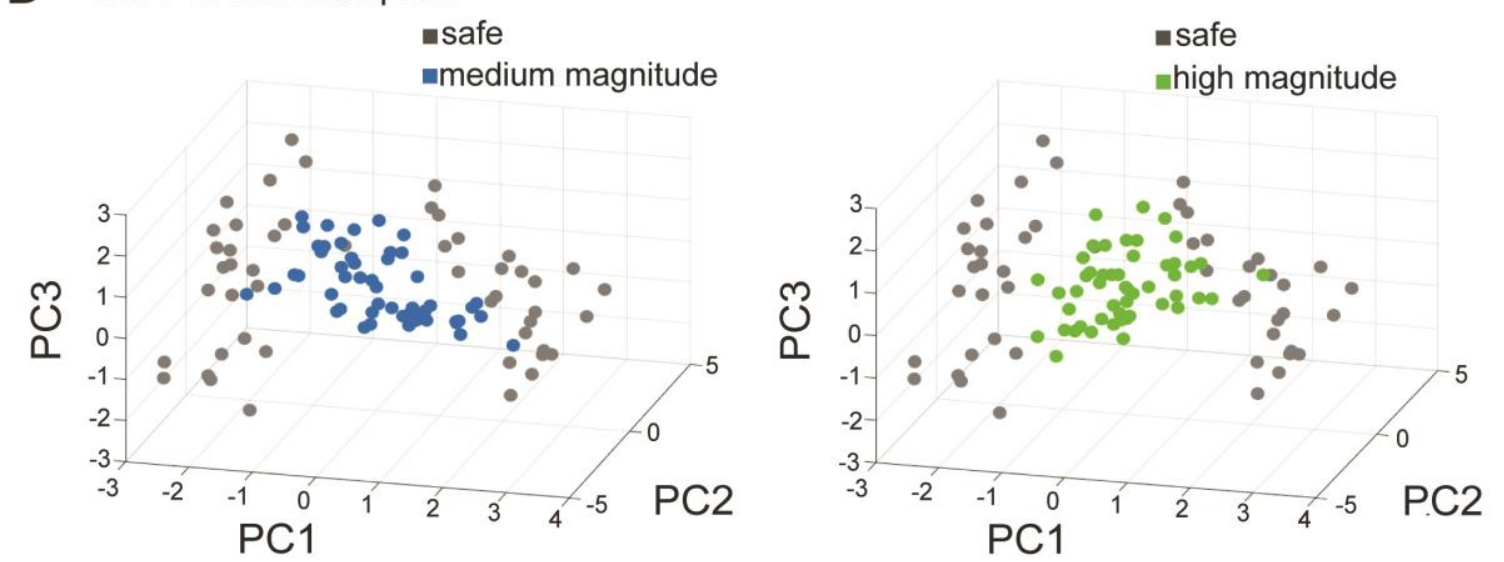

Figure 7. Subspace Alignment between Safe and Equivalent Risky Offers. (A) For an example structure, OFC, shows the explained variance (as a proportion of total variance). The left panel shows the explained variance in both safe (grey) and medium stakes (blue) response by projecting both sets of responses into safe response subspace, due to each of the first 10 principal components. The right panel is similar, except that it shows explained variance by projecting both sets of responses into risky response subspace. (B) same as (A), except that it shows high stakes (green) offer projections into safe subspace (left) and safe (grey) offer projections into high stakes subspace (right). (C) a summary of the difference between the average shuffle alignment index and either the safe-medium (left bar) or the safe-high (right bar) alignment index. Error bars indicate the standard error across computed differences. (D) For an example structure, OFC, left panel: projections of medium stakes (blue) responses and safe (grey) responses, into safe subspace. Right panel: projections of high stakes (green) responses and safe (grey) responses, into safe subspace. 


\section{DISCUSSION}

We examined responses of neurons in six core reward regions to risky and safe offers. By using a large number of risky offers for each neuron, we were able to identify, post-hoc, a subset

540 of risky offers with values equivalent to those of the safe offers. We were then able to ask

541 whether responses to safe and subjective value-matched risky offers were the same, as predicted

542 by common currency models. They were not. Indeed, responses elicited by safe offers were no

543 more similar to those elicited by equally valued risky offers than to any other risky offer in our

544 offer set, indicating that the neural codes used for risky and safe options are unrelated. Moreover,

545 a simple classifier could easily distinguish safe from risky options, even when limited to the

546 subset of neurons most likely to carry a common currency code. Finally, safe and risky options

547 elicited responses in distinct non-collinear subspaces. Together these results provide strong

548 evidence against the idea that the brain makes use of a common currency code for risky and safe

549 offers.

550 Even among those who favor the common currency hypothesis, there is some debate

551 about the most likely region or regions in which common currency coding is likely to occur

552 (Rangel et al., 2008; Kable \& Glimcher, 2009; Padoa-Schioppa, 2011; Rushworth et al., 2011;

553 Wunderlich et al., 2012). One tradition focuses on the OFC and most research in that tradition

554 focuses on area 13 (Tremblay \& Schultz, 1999; Wallis, 2007; Padoa-Schioppa, 2011). Some

555 work also implicates area 11 (Rudebeck \& Murray, 2014). We tested both areas. Another

556 tradition, much of it based on human neuroimaging, favors vmPFC, which is quite different from

557 OFC, both in terms of connectivity and other possible functions (Blair, 2008; Noonan et al.,

558 2011; FitzGerald et al., 2012; reviewed in Levy \& Glimcher, 2012, Bartra et al., 2013, and in

559 Clithero \& Rangel, 2014). The primate homologue of vmPFC is unclear - it may be area 14 or it 
may be area 32 (Myers-Schulz \& Koenigs, 2012; Neubert et al., 2015). We tested both. A third tradition emphasizes the likely importance of PCC for common currency functions (McCoy \&

562 Platt, 2005; Kable \& Glimcher, 2007). Finally, another major theory focuses on the ventral

563 striatum, especially on the NAc core region (Beck et al., 2009; Knutson et al., 2009; Staudinger

564 et al., 2009; Cai et al., 2011; Strait et al., 2015). Our study, which uses the same task in all

565 regions, and finds the same lack of common currency coding, therefore represents a relatively

566 complete list of putative common currency regions, and raises the possibility that such a code

567 does not exist anywhere in the brain. Note, however, that our results do not demonstrate that

568 these areas have identical functions; instead they indicate that whatever value signals are

569 available in these regions are not amodal in our risky choice task.

570 The idea of a common currency representation argues that there is a single final common

571 pathway for value that can feed into - but is conceptually distinct from - action selection. If the

572 brain does not make use of a common currency, then how can we compare values of dissimilar

573 things? There are many possible answers (Vlaev et al., 2011; Hayden \& Niv, 2020). Process

574 models of choice that eschew value representations include heuristic approaches, sampling-based

575 approaches, and embodied/premotor theories. They also include, for example, distributed choice

576 implementations such as that of bee and ant swarms (Seeley et al., 1991; Marshall et al., 2009;

577 Seeley et al., 2012; Bose et al., 2017; Pirrone et al., 2018). In bee swarms, no single bee has

578 access to the value of an option on a universal scale; instead the comparison is made in an

579 indirect manner (Seeley et al., 1991; Seeley et al., 2012). What these approaches have in

580 common is that they do not involve direct comparison of values; instead, they achieve choice by

581 indirect manners. Such approaches tend to be well tailored to natural decision-making contexts in

582 which single options often appear, and the decision must occur without knowledge of the values 
583 of the alternatives (Hayden, 2017). In any case, common currency is one of several possible

584 ways to describe the patterns of choice observed in human and non-human animal decision-

585 makers.

Previous results have demonstrated amply that neurons in the brain have responses that

587 correlate with the values of offers that differ in the ratio of their components (e.g. Padoa-

588 Schioppa \& Assad, 2006). For example, we have shown that two matched risky options, high-

589 probability low stakes and low-probability high stakes, if equally valued, produced responses

590 along a value axis in vmPFC and VS (Strait et al., 2014; Strait et al., 2015). Such responses

591 satisfy one prediction of the common currency hypothesis. Our new results presented here do not

592 vitiate these earlier ones; instead, they demonstrate the limitation of past findings - that they did

593 not test enough conditions to fully falsify the common currency theory. Specifically, our current

594 results suggest that a second criterion for common currency coding, a common code for options

595 that differ in kind, not just in ratio, is not satisfied in any of the major proposed reward regions.

596 These results, then, suggest that key reward areas can integrate across dimensions using a single

597 scale but may use different scales for qualitatively different offer types. Note that, in a trivial

598 sense, risky and safe options are different ratios of risk and stakes - but it is clear they differ

599 psychologically. For example, the risky option affords an opportunity for learning/adjustment,

600 while the safe does not. The risky option may trigger different processes, such as anxiety or

601 savoring (Lopes, 1987; Loewenstein et al., 2001).

602 Perhaps the most intriguing result is our finding that different equally valued stimuli are

603 encoded in non-collinear response subspaces. The brain can make use of response subspaces to

604 keep pieces of information separate. For example, premotor plans can be kept in an output-null

605 subspace so that motor planning can occur without risking triggering a premature action 
606 (Kaufman et al., 2014; Elsayed et al., 2016). We have recently explored the idea that subspace

607 orthogonalization in reward regions may also be used to sequester evaluation from comparison in

608 time (Yoo and Hayden, 2020). Our results suggest that subspace orthogonalization may have a

609 second benefit for reward regions - in particular, that it can segregate qualitatively different

610 option types (Semedo et al., 2019; Stokes et al., 2020). That in turn may serve a categorization

611 function - that is, it may help the brain identify, to downstream decoders, which stimulus type it

612 is encoding. The decoder, then, would have the ability to know whether the stimulus presented

613 was risky or safe, and which decoding procedure to use. This idea, however, is speculative, and

614 further research will be required to test it.

615 Our work relates to a major ongoing debate about the function of OFC, a set of regions

616 whose functions have long been discussed (reviewed in Gardner \& Schoenbaum, 2020). One

617 prominent theory links it to value-specific functions, most notably in representing the values of

618 options (Wallis, 2007; Padoa-Schioppa, 2011; Rudebeck \& Murray, 2014). However, another

619 theory holds that it serves primarily to implement expectancy signalling - that is, it encodes the

620 properties of potential outcomes associated with reward (reviewed in Gardner \& Schoenbaum,

621 2020). These outcomes may include the reward value but would include all other features. For

622 this reason, qualitatively different offers that have the same value should, according to the

623 expectancy theory, produce unrelated neural responses (Wang \& Hayden, 2017). The expectancy

624 theory, more generally, is associated with the idea that the function of OFC is to implement a

625 cognitive map of task space (Wilson et al., 2014; Shuck et al., 2016; Behrens et al., 2018; Schuck

$626 \&$ Niv, 2019). Our results, then, not only endorse the expectancy and cognitive map theories of

627 OFC function, but suggest they may apply to other putative reward regions as well. 


\section{References}

Azab, H. and Hayden, B. Y. (2017). Correlates of decisional dynamics in the dorsal anterior cingulate cortex. PLoS Biology. https://doi.org/10.1371/journal.pbio.2003091

Azab, H. and Hayden, B. Y. (2018). Correlates of economic decisions in the dorsal and subgenual anterior cingulate cortices. European Journal of Neuroscience. https://doi.org/10.1111/ejn.13865

Azab, H. and Hayden, B. Y. (2020). Partial integration of the components of value in anterior cingulate cortex. Behavioral Neuroscience, 134, 296-308.

Barseghyan, L., Molinari, F., O'Donoghue, T., and Teitelbaum, J. C. (2013). The nature of risk preferences: Evidence from insurance choices. American Economic Review, 103. analysis of BOLD fMRI experiments examining neural correlates of subjective value.

642 Beck, A., Schalgenhauf, F., Wüstenberg, T., Hein, J., Kienast, T., ... and Wrase, J. (2009). Ventral striatal activation during reward anticipation correlates with impulsivity in

645 Behrens, T. E. J., Muller, T. H., Wittington, J. C. R., Mark, S. Baram, A. B. ... and KurthNelson, Z. (2018). What is a cognitive map? Organizing knowledge for flexible behavior.

648 Blair, R. J. R. (2008). The amygdala and ventromedial prefrontal cortex: functional contributions and dysfunction in psychopathy. Phil. Trans. R. Soc. B, 363, 2557-2565

650 Blanchard, T. C., Hayden, B. Y., and Bromber-Martin, E. S. (2015). Orbitofrontal cortex uses 
distinct codes for different choice attributes in decisions motivated by curiosity. Neuron, $85,602-614$.

Bose, T., Reina, A., and Marshall, J. A. R. (2017). Collective decision-making. Current Opinion in Behavioral Sciences, 16.

Cai, X., Kim, S., and Lee, D. (2011). Heterogeneous coding of temporally discounted values in the dorsal and ventral striatum during intertemporal choice. Neuron, 69, 170-192.

657 Clithero, J. A. and Rangel, A. (2014). Informatic parcellation of the network involved in the computation of subjective value. Social cognitive and affective neuroscience, 9, 12891302.

Dorris, M. C. and Glimcher, P. W. (2004). Activity in posterior parietal cortex is correlated with the relative subjective desirability of action. Neuron, 44 . cortex. Nature Communications, 7.

Farashahi, S., Azab, H., Hayden, B., and Soltani, A. (2018). On the flexibility of basic risk attitudes in monkeys. Journal of Neuroscience.

668 Farashahi, S., Donahue, C. H., Hayden, B. Y., Lee, D., and Soltani, A. (2019). Flexible combination of reward information across primates. Nat. Hum. Behav., 3, 1215-1224.

670 Farovik, A., Place, R. J., McKenzie, S., Porter, B., Munro, C. E., and Eichenbaum., H. (2015). contexts that guide memory retrieval. The Journal of Neuroscience, 35, 8333-8344.

673 FitzGerald, T. H. B., Friston, K. J., and Dolan, R. J. (2012). Action-specific value signals in 
reward-related regions of the human brain. The Journal of Neuroscience, 32, 16417-

675

676

677

678

679

680

681

682

683

684

685

686

687

688

689

690

691

692

693

694

695

696

16423.

FitzGerald, T. H. B., Seymour, B., Dolan, R. J. (2009). The role of human orbitofrontal cortex in value comparison for incommensurable objects. The Journal of Neuroscience, $29,8388-8395$.

Gallego, J. A., Perich, M. G., Miller, L. E., and Solla, S. A. (2017). Neural manifolds for the control of movement. Neuron, 94.

Gardner, M. P. H. and Schoenbaum, G. (2020). The orbitofrontal cartographer. Psyarxiv.

Glimcher, P. W., Dorris, M. C., and Bayer, H. M. (2005). Physiological utility theory and the neuroeconomics of choice. Games and Economic Behavior, 52, 213-256.

Gross, J., Woelbert, E., Zimmermann, J., Okamoto-Barth, S., Riedl, A. and Goebel, R. (2014). Value signals in the prefrontal cortex predict individual preferences across reward categories. Journal of Neuroscience, 34, 7580-7586.

Hayden, B. Y. (2017). Economic choice: The foraging perspective. Current Opinion in Behavioral Sciences, 24, 1-6.

Hayden, B., Heilbronner, S., and Platt, M. (2010). Ambiguity aversion in rhesus macaques. Frontiers in Neuroscience, 4.

Hayden, B. Y. and Niv, Y. (2020). The case against economic values in the brain. PsyArXiv.

Heilbronner, S. R. (2017). Modeling risky decision-making in nonhuman animals: shared core features. Current Opinion in Behavioral Sciences. https://doi.org/10.1016/j.cobeha.2017.03.001

Heilbronner, S. R. and Hayden, B. Y. (2013). Contextual factors explain risk-seeking preferences in rhesus monkeys. Frontiers in Neuroscience. https://doi.org/10.3389/fnins.2013.00007 
Kable, J. K. and Glimcher, P. W. (2007). The neural correlates of subjective value during intertemporal choice. Nature Neuroscience, 10(12).

Kable, J. W. and Glimcher, P. W. (2009). The neurobiology of decision: Consensus and controversy. Neuron, 63, 733-745.

Kahnt, T., Heinzle, J., Park, S. Q., and Haynes, J-D. (2010). The neural code of reward anticipation in human orbitofrontal cortex. Proceedings of the National Academy of Sciences, 107, 6010-6015.

Kaufman, M. T., Churchland, M. M., Ryu, S. I., and Shenoy, K. V. (2015). Vacillation, indecision and hesitation in moment-by-moment decoding of monkey motor cortex. eLife. doi: 10.7554/eLife.04677.001

Kennerley, S. W. \& Wallis, J. D. (2009). Reward-dependent modulation of working memory in 689 lateral prefrontal cortex. Journal of Neuroscience, 29(10).

Kim, S., Bobeica, I., Gamo, N. J., Arnsten, A. F. T., and Lee, D. (2012). Effects of $\alpha-2$ A adrenergic receptor agonist on time and risk preference in primates. Psychopharmacology, 219, 363-375.

712 Klein, J. T., Deaner, R. O., and Platt, M. L. (2008). Neural correlates of social target value in macaque parietal cortex. Current Biology, 18, 419-424.

714 Knutson, B., Delgado, M. R., and Phillips, P. E. M. (2009). Chapter 25 - Representation of subjective value in the striatum. Neuroeconomics, Academic Press, 389-406. Glimcher,

717 Krajbich, I., Armel, C., and Rangel A. (2010). Visual fixations and the computation and comparison of value in simple choice. Nature Neuroscience, 13, 1292-1298.

719 Lau, B. and Glimcher, P. W. (2008). Value representations in the primate striatum during 
matching behavior. Neuron, 58, 451-463.

721 Levy, D. J. and Glimcher, P. W. (2012). The root of all value: A neural common currency for choice. Current Opinion in Neurobiology. https://doi.org/10.1016/j.conb.2012.06.001

723 Lopes, L. L. (1987). Between hope and fear: The psychology of risk. Advances in sExperimental Social Psychology. https://doi.org/10.1016/S0065-2601(08)60416-5

725 Loewenstein, G. F., Weber, E. U., Hsee, C. K., and Welch, N. (2001). Risk as feelings. Psychological Bulletin, 127.

Lucantonio, F., Gardner, M. P. H., Mirenzi, A., Nerman, L. E., Takahashi, Y. K., and Schoenbaum, G. (2015). Neural estimates of imagined outcomes in basolateral amygdala depend on orbitofrontal cortex. Journal of Neuroscience, 35, 16521-16530.

Maisson, D. J., Cash-padgett, T. V, and Hayden, B. Y. (2020). A functional hierarchy for choice in medial prefrontal cortex. BioRxiv.

Marshall, J. A. R., Bogacz, R., Dornhaus, A., Planque, R., Kovacs, T., and Franks, N. R. (2009). Society, Interface. 61065-1074

McCoy, A. N. and Platt, M. L. (2005). Risky-sensitive neurons in macaque posterior cingulate cortex. Nature Neuroscience, 8.

737 McGinty, V. B., Rangel, A., \& Newsome, W. T. (2016). Orbitofrontal cortex value signals depend on fixation location during free viewing. Neuron, 90, 1299-1311.

742 Montague, P. R. and Berns, G. S. (2002). Neural economics and the biological substrates of 
valuation. Neuron. https://doi.org/10.1016/S0896-6273(02)00974-1

744 Myers-Schulz, B. and Koenigs, M. (2012). Functional anatomy of ventromedial prefrontal cortex: implications for mood and anxiety disorders. Molecular Psychiatry, 17, 132-141.

746 Neubert, F-X., Mars, R. B., Sallet, J., and Rushworth, M. F. S. (2015). Connectivity reveals relationship of brain areas for reward-guided learning and decision making in human and monkey frontal cortex. PNAS, 112, E2695-E2704.

749 Noonan, M. P., Mars, R. B., and Rushworth, M. F. S. (2011). Distinct roles of three frontal cortical areas in reward-guided behavior. Journal of neuroscience, 31, 143999-14412.

751 O'Doherty, J., Kringelbach, M., Rolls, E. et al. (2001). Abstract reward and punishment representations in the human orbitofrontal cortex. Nature Neuroscience, 4, 95-102.

753 O'Donoghue, Ted, and Matthew Rabin. 2015. Present bias: Lessons learned and to be learned. $754 \quad$ American Economic Review, 105.

755 Oşan R., Zhu, L., Shoham, S., and Tsien, J. Z. (2007) Subspace projection approaches to classification and visualization of neural network-level encoding patterns. PLOS ONE, 2.

757 Padoa-Schioppa, C. (2011). Neurobiology of economic choice: A good-based model. Annual $758 \quad$ Review of Neuroscience, 3.

759 Padoa-Schioppa, C. and Assad, J. A. (2006). Neurons in the orbitofrontal cortex encode economic value. Nature. https://doi.org/10.1038/nature04676 Padoa-Schioppa, C. and Conen, K. E. (2017). Orbitofrontal cortex: A neural circuit for

763 Padoa-Schioppa, C. and Schoenbaum, G. (2015). Dialogue on economic choice, learning theory, and neuronal representations. Current Opinion in Behavioral Sciences.

$765 \quad$ https://doi.org/10.1016/j.cobeha.2015.06.004 
Paxinos, G., Petrides, M., Huang, X., \& Toga, A. W. (2008). The rhesus monkey brain in stereotaxic coordinates. Elsvier Science.

Pirrone, A., Azab, H., Hayden, B. Y., Stafford, T., \& Marshall, J. A. R. (2018). Evidence for the speed-value trade-off: Human and monkey decision making is magnitude sensitive. Decision, 5(2), 129-142.

Platt, M. L. and Huettel, S. A. (2008). Risky business: The neuroeconomics of decision making under uncertainty. Nature Neuroscience, 11 .

Rangel, A., Camerer, C., and Montague, P. (2008). A framework for studying the neurobiology of value-based decision making. Nature Review Neuroscience, 9, 545-556.

Rich, E. and Wallis, J. (2016). Decoding subjective decisions from orbitofrontal cortex. Nature Neuroscience, 19, 973-980.

777 Rolls, E. T. (2000). The orbitofrontal cortex and reward. Cerebral Cortex, 10, 284-294.

778 Rudebeck, P. H. and Murray, E. A. (2014). The orbitofrontal oracle: Cortical mechanisms for the prediction and evaluation of specific behavioral outcomes. Urology.

781 Rudebeck, P. H., Saunders, R. C., Lundgren, D. A., and Murray, E. A. (2017). Specialized representations of value in the orbital and ventrolateral prefrontal cortex: Desirability

784 Rushworth, M. F. S., Noonan, M. A. P., Boorman, E. D., Walton, M. E., and Behrens, T. E. (2011). Frontal cortex and reward-guided learning and decision-making. Neuron, 70, 1054-1069. decisions. Journal of Neurophysiology, 114, 1382-1398. 
Schoenbaum, G., Chiba, A. A., \& Gallagher, M. (1998). Orbitofrontal cortex and basolateral amygdala encode expected outcomes during learning. Nature Neuroscience, 1, 155-159.

791 Schoenbaum, G., Setlow, B., Saddoris, M. R., and Gallagher, M. (2003). Encoding predicted outcome and acquired value in orbitofrontal cortex during cue sampling depends upon

Schoenbaum, G., Takahashi, Y., Liu, T. L., and Mcdannald, M. A. (2011). Does the orbitofrontal cortex signal value? Annals of the New York Academy of Sciences. https://doi.org/10.1111/j.1749-6632.2011.06210.x Seeley, T.D., Camazine, S., and Sneyd, J. (1991). Collective decision-making in honey

Seeley, T. D., Visscher, P. K., Schlegel, T., Hogan, P. M., Franks, N. R., and Marshall, J. A. (2012). Stop signals provide cross inhibition in collective decision-making by honeybee swarms. Science, 6, 108-111 interact through a communication subspace. Neuron, 102, 249-259. cortex represents a cognitive map of states space. Neuron, 91, 1402-1412.

806 Schuck, N. W. and Niv, Y. (2019). Sequential replay of nonspatial task states in the human hippocampus. Science, 80(364).

808 Sleezer, B. J., Castagno, M. D., and Hayden, B. Y. (2016). Rule encoding in orbitofrontal cortex and striatum guides selection. Journal of Neuroscience, 36, 11223-11237. saccades with variable reward. Journal of Neurophysiology, 104, 2634-2653. 
812 So, N. Y. and Stuphorn, V. (2016). Supplementary eye field encodes confidence in decisions under risk. Cerebral Cortex, 26, 764-782.

814 Stokes, M., Muhle-Karbe, P. S., Myers, N. E. (2020). Theoretical distinction between functional states in working memory and their corresponding neural states. PsyArXiv.

Strait, C. E., Blanchard, T. C., and Hayden, B. Y. (2014). Reward value comparison via mutual inhibition in ventromedial prefrontal cortex. Neuron, 82 .

Strait, C. E., Sleezer, B., J., and Hayden, B. Y. (2015). Signatures of value comparison in ventral striatum neurons. PLOS Biology, https://doi.org/10.1371/journal.pbio.1002173 expected value and prediction error encoding in the ventral striatum. NeuroImage, 47 ,

Takahashi, Y. K., Roesch, M. R., Wilson, R. C., Toreson, K., O’Donnell, P., Niv, Y., and Schoenbaum, G. (2011). Expectancy-related changes in firing of dopamine neurons

829 Tremblay, L. and Schultz, W. (1999). Relative reward preference in primate orbitofrontal cortex. Nature, 398, 704-708.

831 Vlaev, I., Chater, N., Stewart, N., and Brown, G. D. A. (2011). Does the brain calculate value? Trends in Cognitive Sciences. https://doi.org/10.1016/j.tics.2011.09.008 
835 Wang, M. and Hayden, B. (2017). Reactivation of associative structure specific outcome responses during prospective evaluation in reward-based choices. Nature Communications, 8. https://doi.org/10.1038/ncomms15821

838 Wikenheiser, A. M. and Schoenbaum, G. (2016). Over the river, through the woods: cognitive maps in the hippocampus and orbitofrontal cortex. Nature Reviews Neuroscience, 17, 513-523.

841 Wilson, R. C., Takahashi, Y. K., Schoenbaum, G., and Niv, Y. (2014). Orbitofrontal cortex as a cognitive map of task space. Neuron, 81, 267-279.

843 Wunderlich, K., Dayan, P., and Dolan, R. (2012). Mapping value based planning and extensively

844 trained choice in the human brain. Nature Neuroscience, 15, 786-791.

845 Xie, J. and Padoa-Schioppa, C. (2016). Neuronal remapping and circuit persistence in economic decisions. Nature Neuroscience, 19, 855-861. Xie, Y., Nie, C., \& Yang, T. (2018). Covert shift of attention modulates the value

849 Yoo, S. B. M. and Hayden, B. Y. (2018). Economic choice as an untangling of options into actions. Neuron, 99(3), 434-447. https://doi.org/10.1016/j.neuron.2018.06.038

851 Yoo, S. B. M. and Hayden, B. Y. (2020). The transition from evaluation to selection involves 852 neural subspace reorganization in core reward regions. Neuron, 105. 


\section{METHODS}

859 Health Service's Guide for the Care and Use of Animals. Six male rhesus macaques (Macaca

860 mulatta) served as subjects. A small prosthesis head fixation was used. Animals were habituated

861 to laboratory conditions and then trained to perform oculomotor tasks for liquid rewards. We

862 place a Cilux recording chamber (Crist Instruments) over the area of interest (see Behavioral

863 tasks for breakdown). We verified positioning by magnetic resonance imaging with the aid of a

864 Brainsight system (Rogue Research). Animals received appropriate analgesics and antibiotics

865 after all procedures. Throughout both behavioral and physiological recording sessions, we kept

866 the chamber with regular antibiotic washes and we sealed them with sterile caps.

868 Instruments) guided by a micromanipulator (NAN Instruments). All recording sites were selected

869 based on the boundaries given in the Paxinos atlas (Paxinos et al., 2008). In all cases we sampled

870 evenly across the regions. Neuronal recordings in OFC were collected from subjects $P$ and $S$;

871 recordings in rOFC were collected from subjects $V$ and $P$; recordings in vmPFC were collected

872 from subjects $B$ and $H$; recordings in pgACC were collected from subject $B$ and $V$; recordings

873 from PCC were collected from subject $P$ and $S$; and recording in VS were collected from subject

$874 B$ and $C$. Specifically (see Figure 1B):

875 We defined rOFC 11 as lying within the coronal planes situated between 34.05 and

$87642.15 \mathrm{~mm}$ rostral to the interaural plane, the horizontal planes situated between 4.5 and $9.5 \mathrm{~mm}$ 
877 from the brain's ventral surface, and the sagittal planes between 3 and $14 \mathrm{~mm}$ from the medial

878 wall. The coordinates correspond to area 11 in Paxinos et al. (2008). $\mathrm{mm}$ rostral to the interaural plane, the horizontal planes situated between 3 and $6.5 \mathrm{~mm}$ from the brain's ventral surface, and the sagittal planes between 5 and $14 \mathrm{~mm}$ from the medial wall. The coordinates correspond to area $13 \mathrm{~m}$ in Paxinos et al. (2008).

We defined vmPFC 14 as lying within the coronal planes situated between 29 and 44

$884 \mathrm{~mm}$ rostral to the interaural plane, the horizontal planes situated between 0 and $9 \mathrm{~mm}$ from the

885 brain's ventral surface, and the sagittal planes between 0 and $8 \mathrm{~mm}$ from the medial wall. These coordinates correspond to area $14 \mathrm{~m}$ in Paxinos et al. (2008).

We defined pgACC 32 as lying with the coronal planes situated between 30.90 and 40.10 $\mathrm{mm}$ rostral to the interaural plane, the horizontal planes situated between 7.30 and $15.50 \mathrm{~mm}$

889 from the brain's dorsal surface, and the sagittal planes between 0 and $4.5 \mathrm{~mm}$ from the medial 890 wall (Figure 1B). Our recordings were made from central regions within these zones, which

891 correspond to area 32 in Paxinos et al. (2008).

892 We defined PCC 29/31 as lying within the coronal planes situated between $2.88 \mathrm{~mm}$

893 caudal and $15.6 \mathrm{~mm}$ rostral to the interaural plane, the horizontal planes situated between 16.5

894 and $22.5 \mathrm{~mm}$ from the brain's dorsal surface, and the sagittal planes between 0 and $6 \mathrm{~mm}$ from

895 the medial wall. The coordinates correspond to area 29/31 in Paxinos et al. (2008).

We defined VS as lying within the coronal planes situated between 20.66 and $28.02 \mathrm{~mm}$

897 rostral to the interaural plane, the horizontal planes situated between 0 and $8.01 \mathrm{~mm}$ from the

898 ventral surface of the striatum, and the sagittal planes between 0 and $8.69 \mathrm{~mm}$ from the medial

899 wall. Note that our recording sites were targeted towards the nucleus accumbens core region of 
the VS.

We confirmed recording location before each recording session using our Brainsight system with structural magnetic resonance images taken before the experiment. Neuroimaging was performed at the Rochester Center for Brain Imaging on a Siemens 3T MAGNETOM Trio

904 Tim using $0.5 \mathrm{~mm}$ voxels. We confirmed recording locations by listening for characteristic

905 sounds of white and gray matter during recording, which in all cases matched the loci indicated

906 by the Brainsight system with an error of $\sim 1 \mathrm{~mm}$ in the horizontal plane and $\sim 2 \mathrm{~mm}$ in the $\mathrm{z}-$ 907 direction.

908 Electrophysiological techniques. Either single (FHC) or multi-contact electrodes (V-

909 Probe, Plexon) were lowered using a microdrive (NAN Instruments) until waveforms between

910 one and three neuron(s) were isolated. Individual action potentials were isolated on a Plexon

911 system (Plexon, Dallas, TX) or Ripple Neuro (Salt Lake City, UT). Neurons were selected for

912 study solely on the basis of the quality of isolation; we never preselected based on task-related

913 response properties. All collected neurons for which we managed to obtain at least 300 trials

914 were analyzed; no neurons that surpassed our isolation criteria were excluded from analysis.

$915 \quad$ Eye-tracking and reward delivery. Eye position was sampled at 1,000 $\mathrm{Hz}$ by an infrared

916 eye-monitoring camera system (SR Research). Stimuli were controlled by a computer running

917 Matlab (Mathworks) with Psychtoolbox and Eyelink Toolbox. Visual stimuli were colored

918 rectangles on a computer monitor placed $57 \mathrm{~cm}$ from the animal and centered on its eyes (Fig.

919 1A). A standard solenoid valve controlled the duration of juice delivery. Solenoid calibration was

920 performed daily.

921 Behavioral tasks. Six monkeys performed in the risky choice task. Both tasks made use

922 of vertical rectangles indicating reward amount and probability. We have shown in a variety of 
923 contexts that this method provides reliable communication of abstract concepts such as reward,

924 probability, delay, and rule to monkeys (Blanchard et al., 2015; Sleezer et al., 2016; Mehta et al., 925 2019).

Risky choice task. . The task presented two offers on each trial. A rectangle 300 pixels tall

927 and 80 pixels wide represented each offer $\left(11.35^{\circ}\right.$ of visual angle tall and $4.08^{\circ}$ of visual angle

928 wide; Fig. 2A). Two parameters defined gamble offers, stakes and probability. Each gamble

929 rectangle was divided into two portions, one red and the other either gray, blue, or green. The

930 size of the color portions signified the probability of winning a small (125 $\mu 1$, gray), medium

931 (165 $\mu$ l, blue), or large reward ( $240 \mu$, green), respectively. We used a uniform distribution

932 between 0 and $100 \%$ for probabilities. The size of the red portion indicated the probability of no

933 reward. Offer types were selected at random with a $43.75 \%$ probability of blue (medium

934 magnitude) gamble, a $43.75 \%$ probability of green (high magnitude) gambles, and a $12.5 \%$

935 probability of gray options (safe offers).

936 On each trial, one offer appeared on the left side of the screen and the other appeared on

937 the right. We randomized the sides of the first and second offer. Both offers appeared for $400 \mathrm{~ms}$

938 and were followed by a 600-ms blank period. After the offers were presented separately, a

939 central fixation spot appeared and the monkey fixated on it for $100 \mathrm{~ms}$. Following this, both

940 offers appeared simultaneously and the animal indicated its choice by shifting gaze to its

941 preferred offer and maintaining fixation on it for $200 \mathrm{~ms}$. Failure to maintain gaze for $200 \mathrm{~ms}$ did

942 not lead to the end of the trial but instead returned the monkey to a choice state; thus monkeys

943 were free to change their mind if they did so within $200 \mathrm{~ms}$ (although in our observations, they

944 seldom did so). Following a successful 200-ms fixation, the gamble was resolved and the reward

945 was delivered. We defined trials that took $>7 \mathrm{sec}$ as inattentive trials and we did not include 
946 them in the analyses (this removed $\sim 1 \%$ of trials). Outcomes that yielded rewards were

947 accompanied by a visual cue: a white circle in the center of the chosen offer. All trials were

948 followed by an 800-ms intertrial interval with a blank screen.

949 Estimation of subjective value equivalence. We calculated the indifference point between

950 safe and risky offers. For each subject, independently, we fitted a sigmoidal function to the

951 distribution of choices (safe or risky) across the full range of risky offer probabilities.

$$
f(x)=\frac{a}{b+e^{(b x)}}
$$

where $\mathrm{x}=$ the probability associated with the risky offer, and $f(\mathrm{x})=$ the likelihood of

954 choosing the safe offer; a (the maximum value of the curve) and b (the growth rate; steepness)

955 are coefficients of the function, estimated by the fitting procedure for maximizing $R^{2}$. Using the

956 fitted sigmoidal function, we then estimated the value of $\mathrm{x}$ needed to produce a safe choice

957 likelihood of 0.5 ; that is, a risky offer choice is equally likely as a safe choice. This is called the

958 indifference point, and it allows us to calculate the risky offer value that is considered by the

959 subject to be of equivalent value to that of the safe offer. We performed this analysis separately

960 for medium and high stakes gambles, and separately for each subject.

962 rasters to the presentation of the first offer and averaging firing rates across multiple trials. We

963 calculated firing rates in 20-ms bins but we analyzed them in longer (500 ms) epochs. Some

964 statistical tests of neuronal activity were only appropriate when applied to single neurons

965 because of variations in response properties across the population.

$966 \quad$ Pure value cell analysis. For each neuron, we separated the firing rates in response to

967 offer 1 by whether they corresponded to a safe or risky offer. Each epoch consisted of a $500 \mathrm{~ms}$ 
968 window, beginning $100 \mathrm{~ms}$ after the onset of the corresponding offer, which we have found in

969 previous work to roughly correspond with the stimulus-response lag time (Strait et al., 2014).We

970 calculated the mean firing rates for each neuron across all safe or risky trials. We then performed

971 a t-test between firing rate vectors in response to safe and risky offers with equivalent value. We

972 calculated the remaining proportion of total neurons in a given structure after neglecting those

973 with significantly different firing rates in response to equal offers. We then repeated this analysis

974 for firing rates in response to safe offers and a randomly chosen risky offer. We repeated this

975 control step 1000 times. The proportion of sample neurons remaining after accounting for those

976 with statistically differing responses constituted the true positive rate, while the average

977 proportion across the 1000 random samples constituted the false positive rate, or the rate at

978 which we should expect to see statistically similar firing rates purely by chance. To confirm

979 statistical significance, we determined the proportion of bootstrapped false positive rates that

980 were less than the true positive rate. A statistically significant true positive rate would be larger

981 than at least 950 of the 1000 bootstrapped samples. Therefore, the $p$-value is equal to the

982 proportion of false positive rates that are larger than true positive rates (or, the difference

983 between one and that the number of false positive rates that are smaller than the true positive

984 rate). That is, the larger the true positive rate is relative to the 1000 samples of false positive

985 rates, the smaller the $p$-value will get.

987 response to offer 1 by whether they corresponded to a safe or risky offer. We calculated the mean

988 firing rates for each neuron across all trials safe and risky trials. We then computed the absolute

989 value of the difference between mean firing rates in response to safe and equivalent risky offers.

990 To determine significance, we performed a 1000-sample bootstrap of randomly selected risky 
991 offers, computed the delta, ranked the deltas in ascending order and asked how many of these

9921000 control deltas were less than the true delta. We next defined subsets of neurons ranging

993 from $1-100 \%$ of all recorded neurons in a structure. We performed these same calculations, and

994 ordered the differences from least to greatest. For each subset, we took the corresponding

995 number of neurons with the smallest response difference (i.e. the best subset) and compared them

996 to a control population (calculated the same was as described previously). We then performed a

997 Komolgorov-Smirnov test to compare the average response difference to equal offers across all

998 subset sizes with the response difference to random offers.

999 Decoding analysis. We built a pseudo-population of pseudo-trials. First, for each epoch,

1000 we isolated firing rate responses to the safe offers and the equivalent risky offers. Then, we

1001 collapsed the firing rates for each trial into an average for the $500 \mathrm{~ms}$ period. We randomly

1002 selected 1000 samples for each neuron, under both risk conditions, resulting in two $n$ X 1000

1003 matrices (one for each label level), where $\mathrm{n}$ represented the number of neurons recorded from

1004 each region. This constituted the pseudo-population of pseudo-trials. To execute the decoder,

1005 each matrix was split in half and concatenated with the half from the other label. We used one of

1006 these matrices to train a binary support vector machine, the other was used for cross-validation.

1007 We used the trained model to predict the binary label (safe or risky) for each pseudo-trial in the

1008 cross-validation set. We then compared the predicted label to the known label and an accuracy

1009 rate was calculated across predictions. This process was repeated 1000 times for each structure

1010 and epoch to get a distribution of accuracy rates. Thus, the standard error of the mean, used in

1011 displaying the error bars, represents the standard error over the variance of the cross-validations.

1012 Additionally, the exact process was repeated on randomly shuffled data, to confirm that expected

1013 prediction accuracy was $50 \%$ when randomized. 
1015 Specifically, for each structure, we separated offers and neural responses by their risk profile

1016 (safe and equivalent risky offer of medium and high magnitudes), as described previously. For

1017 each neuron, we identified two factors to incorporate into a single condition: time and offer

1018 position. Time included the same $500 \mathrm{~ms}$ period following the onset of offer 1 and preceding the

1019 onset of offer 2. Time was segmented into $20 \mathrm{~ms}$ bins. For each $20 \mathrm{~ms}$ bin, we computed the

1020 mean firing rate across trials on which the offer was positioned on either the left or right of the

1021 screen. Thus, we constructed a condition (time $\mathrm{X}$ offer position) X neuron matrix of mean firing

1022 rates; that is, a $50 \mathrm{X}$ n-neurons matrix. One such matrix was constructed for safe offers, one for

1023 medium, and one for high magnitude risky offers of equivalent value. Firing rates in prefrontal

1024 areas of macaques tend to be sparse. So, we smoothed these matrices using a gaussian filter, with

1025 a sigma equal to one. We then normalized the smoothed matrices, by computing the z-score

1026 within each cell, to account for differences in encoding scaling between neurons.

Next, we performed a principal component analysis, using eigenvalue decomposition, on

1028 the safe response matrix, providing a transformation matrix into which we projected both the

1029 safe response matrix and each of the risky response matrices. We computed the explained

1030 variance due to each of the principal components. We performed the same process of

1031 dimensionality reduction for each of the risky offers, projecting both the safe response and

1032 corresponding risky response data into the resulting principal component spaces (medium

1033 magnitude and high magnitude risky response each into their own principal component spaces).

1034 To determine if the subspaces were aligned, we computed an alignment index:

$$
A_{i d x}=\frac{\operatorname{Tr}\left(D_{\text {risky }}^{T} C_{\text {safe }} D_{\text {risky }}\right)}{\sum_{i=1}^{\text {sel-dim }} \sigma_{\text {safe }}(i)}
$$


1036 where $\operatorname{Tr}()$ is the sum along the diagonal entry, sel-dim = the number of selected principal

1037 components (or ten, in the current study), $\mathrm{D}_{\text {risky }}$ are the set of top sel-dim eigenvectors, $\mathrm{C}_{\text {safe }}$ is the

1038 covariance matrix for the safe responses, $\sigma_{\text {safe }}(i)$ is $\mathrm{i}$-th singular value of $\mathrm{C}_{\text {safe. }}$ Essentially, the

1039 variance explained in safe response by the top ten principal components of the risky responses is

1040 normalized against the sum of the variance explained by the top ten principal components of the

1041 safe responses. Note that we also performed this calculation using both the top 4 and top 7

1042 principal components. This control did not change the results of the significance tests, and so

1043 they are not reported.

To determine the significance of the alignment index we performed a shuffle procedure.

1045 We assume, in this procedure, that neural responses adhere to a fixed correlation structure

1046 (Elsayed et al., 2016). Thus, we tested whether the safe and risky subspaces were more or less

1047 orthogonal, relative to randomly sampling within the space of this fixed correlation structure. We

1048 concatenated all safe and risky offer data into a single matrix. We then computed the covariance

1049 matrix, and performed an eigenvalue decomposition for the covariance matrix. We then

1050 randomly sampled subspaces that were aligned to the fixed correlation structure of the response

1051 space, using a method described by Elsayed et al. (2016), as follows:

1052

$$
v_{\text {align }}=\operatorname{orth}\left(\frac{U \sqrt{S v}}{\|U \sqrt{S v}\|_{2}}\right)
$$

1053 where $\mathrm{U}$ and $\mathrm{S}$ are the eigenvectors and eigenvalue matrices, respectively, of the computed

1054 covariance matrix. A matrix (v) was drawn from a normal distribution with a mean $=0.0$ and

1055 variance $=1.0$. Orth () computes the orthonormal basis of the projected matrix. This process

1056 essentially maintains the neuronal covariance structure of the original covariance matrix used for

1057 the eigenvalue decomposition. We repeated this process across 1000 iterations and computed the 
1058 alignment index for each, according to the above description. We then calculated the average

1059 alignment index and the $95 \%$ confidence intervals across the 1000 iterations.

1060 Multinomial Logistic Regression. To follow-up on the alignment index analysis, we used

1061 the projections of safe, medium, and high stakes risky offers into safe subspace. We selected the

1062 top 10 results principal components, consistent with the alignment index and constituting no less

1063 than $80 \%$ of the total explained variance. We then built a multinomial logistic regression model,

1064 in which projections onto the first 10 principal components, from of the offer types, were used as

1065 simultaneous predictors. The model then set these factors as predictors of a categorical class

1066 variable, labeling each offer type. The resulting model was then tested for significance using the

1067 standard F-statistic for regressions, against an alpha of 0.05. 